\title{
Multiplicity and pseudorapidity distributions of charged particles and photons at forward pseudorapidity in Au+Au collisons at $\sqrt{s_{N N}}=62.4 \mathrm{GeV}$
}

J. Adams, ${ }^{3}$ M. M. Aggarwal, ${ }^{29}$ Z. Ahammed, ${ }^{44}$ J. Amonett, ${ }^{20}$ B. D. Anderson, ${ }^{20}$ D. Arkhipkin, ${ }^{13}$ G. S. Averichev, ${ }^{12}$ S. K. Badyal, ${ }^{19}$ Y. Bai, ${ }^{27}$ J. Balewski, ${ }^{17}$ O. Barannikova, ${ }^{32}$ L. S. Barnby, ${ }^{3}$ J. Baudot, ${ }^{18}$ S. Bekele, ${ }^{28}$ V. V. Belaga, ${ }^{12}$ A. Bellingeri-Laurikainen, ${ }^{39}$ R. Bellwied, ${ }^{47}$ J. Berger, ${ }^{14}$ B. I. Bezverkhny, ${ }^{49}$ S. Bharadwaj, ${ }^{34}$ A. Bhasin, ${ }^{19}$ A. K. Bhati, ${ }^{29}$ H. Bichsel, ${ }^{46}$ J. Bielcik, ${ }^{49}$ J. Bielcikova, ${ }^{49}$ A. Billmeier, ${ }^{47}$ L. C. Bland, ${ }^{4}$ C. O. Blyth, ${ }^{3}$ S.-L. Blyth, ${ }^{21}$ B. E. Bonner, ${ }^{35}$ M. Botje,${ }^{27}$

A. Boucham, ${ }^{39}$ J. Bouchet, ${ }^{39}$ A. V. Brandin, ${ }^{25}$ A. Bravar, ${ }^{4}$ M. Bystersky, ${ }^{11}$ R. V. Cadman, ${ }^{1}$ X. Z. Cai, ${ }^{38}$ H. Caines, ${ }^{49}$ M. Calderón de la Barca Sánchez, ${ }^{17}$ J. Castillo, ${ }^{21}$ O. Catu, ${ }^{49}$ D. Cebra, ${ }^{7}$ Z. Chajecki, ${ }^{28}$ P. Chaloupka, ${ }^{11}$ S. Chattopadhyay, ${ }^{44}$ H. F. Chen, ${ }^{37}$ J. H. Chen, ${ }^{38}$ Y. Chen, ${ }^{8}$ J. Cheng, ${ }^{42}$ M. Cherney, ${ }^{10}$ A. Chikanian, ${ }^{49}$ H. A. Choi,${ }^{33}$ W. Christie, ${ }^{4}$ J. P. Coffin, ${ }^{18}$ T. M. Cormier, ${ }^{47}$ M. R. Cosentino, ${ }^{36}$ J. G. Cramer, ${ }^{46}$ H. J. Crawford, ${ }^{6}$ D. Das, ${ }^{44}$ S. Das, ${ }^{44}$ M. Daugherity, ${ }^{41}$ M. M. de Moura ${ }^{36}$ T. G. Dedovich, ${ }^{12}$ M. DePhillips, ${ }^{4}$ A. A. Derevschikov, ${ }^{31}$ L. Didenko, ${ }^{4}$ T. Dietel, ${ }^{14}$ S. M. Dogra, ${ }^{19}$ W. J. Dong, ${ }^{8}$ X. Dong, ${ }^{37}$ J. E. Draper, ${ }^{7}$ F. Du, ${ }^{49}$ V. B. Dunin, ${ }^{12}$ J. C. Dunlop, ${ }^{4}$ M. R. Dutta Majumdar, ${ }^{44}$ V. Eckardt,${ }^{23}$ W. R. Edwards, ${ }^{21}$ L. G. Efimov, ${ }^{12}$ V. Emelianov, ${ }^{25}$ J. Engelage, ${ }^{6}$ G. Eppley, ${ }^{35}$ B. Erazmus, ${ }^{39}$ M. Estienne, ${ }^{39}$ P. Fachini, ${ }^{4}$ J. Faivre, ${ }^{18}$ R. Fatemi, ${ }^{22}$ J. Fedorisin, ${ }^{12}$

K. Filimonov, ${ }^{21}$ P. Filip, ${ }^{11}$ E. Finch, ${ }^{49}$ V. Fine, ${ }^{4}$ Y. Fisyak, ${ }^{4}$ K. S. F. Fornazier, ${ }^{36}$ J. Fu, ${ }^{42}$ C. A. Gagliardi, ${ }^{40}$ L. Gaillard, ${ }^{3}$ J. Gans, ${ }^{49}$ M. S. Ganti, ${ }^{44}$ F. Geurts, ${ }^{35}$ V. Ghazikhanian, ${ }^{8}$ P. Ghosh, ${ }^{44}$ J. E. Gonzalez, ${ }^{8}$ Y. G. Gorbunov, ${ }^{10}$ H. Gos, ${ }^{45}$ O. Grachov, ${ }^{47}$ O. Grebenyuk, ${ }^{27}$ D. Grosnick, ${ }^{43}$ S. M. Guertin, ${ }^{8}$ Y. Guo, ${ }^{47}$ A. Gupta, ${ }^{19}$ N. Gupta, ${ }^{19}$ T. D. Gutierrez, ${ }^{7}$ T. J. Hallman, ${ }^{4}$

A. Hamed, ${ }^{47}$ D. Hardtke, ${ }^{21}$ J. W. Harris, ${ }^{49}$ M. Heinz,${ }^{2}$ T. W. Henry, ${ }^{40}$ S. Hepplemann, ${ }^{30}$ B. Hippolyte, ${ }^{18}$ A. Hirsch, ${ }^{32}$ E. Hjort, ${ }^{21}$

G. W. Hoffmann, ${ }^{41}$ M. J. Horner, ${ }^{21}$ H. Z. Huang, ${ }^{8}$ S. L. Huang, ${ }^{37}$ E. W. Hughes, ${ }^{5}$ T. J. Humanic, ${ }^{28}$ G. Igo, ${ }^{8}$ A. Ishihara, ${ }^{41}$ P. Jacobs, ${ }^{21}$ W. W. Jacobs, ${ }^{17}$ H. Jiang, ${ }^{8}$ P. G. Jones, ${ }^{3}$ E. G. Judd,${ }^{6}$ S. Kabana, ${ }^{2}$ K. Kang,${ }^{42}$ M. Kaplan, ${ }^{9}$ D. Keane, ${ }^{20}$

A. Kechechyan, ${ }^{12}$ V. Yu. Khodyrev, ${ }^{31}$ B. C. Kim, ${ }^{33}$ J. Kiryluk, ${ }^{22}$ A. Kisiel, ${ }^{45}$ E. M. Kislov, ${ }^{12}$ J. Klay, ${ }^{21}$ S. R. Klein, ${ }^{21}$ D. D. Koetke, ${ }^{43}$ T. Kollegger, ${ }^{14}$ M. Kopytine, ${ }^{20}$ L. Kotchenda, ${ }^{25}$ K. L. Kowalik, ${ }^{21}$ M. Kramer, ${ }^{26}$ P. Kravtsov, ${ }^{25}$ V. I. Kravtsov, ${ }^{31}$ K. Krueger, ${ }^{1}$ C. Kuhn, ${ }^{18}$ A. I. Kulikov, ${ }^{12}$ A. Kumar, ${ }^{29}$ R. Kh. Kutuev, ${ }^{13}$ A. A. Kuznetsov, ${ }^{12}$ M. A. C. Lamont, ${ }^{49}$

J. M. Landgraf, ${ }^{4}$ S. Lange, ${ }^{14}$ F. Laue, ${ }^{4}$ J. Lauret, ${ }^{4}$ A. Lebedev, ${ }^{4}$ R. Lednicky, ${ }^{12}$ C.-H. Lee, ${ }^{33}$ S. Lehocka, ${ }^{12}$ M. J. LeVine, ${ }^{4}$ C. $\mathrm{Li}^{37}{ }^{37}$ Q. Li, ${ }^{47}$ Y. Li, ${ }^{42}$ G. Lin, ${ }^{49}$ S. J. Lindenbaum, ${ }^{26}$ M. A. Lisa, ${ }^{28}$ F. Liu, ${ }^{48}$ H. Liu, ${ }^{37}$ J. Liu, ${ }^{35}$ L. Liu, ${ }^{48}$ Q. J. Liu, ${ }^{46}$ Z. Liu, ${ }^{48}$

T. Ljubicic, ${ }^{4}$ W. J. Llope, ${ }^{35}$ H. Long, ${ }^{8}$ R. S. Longacre, ${ }^{4}$ M. Lopez-Noriega, ${ }^{28}$ W. A. Love, ${ }^{4}$ Y. Lu, ${ }^{48}$ T. Ludlam, ${ }^{4}$ D. Lynn, ${ }^{4}$

G. L. Ma, ${ }^{38}$ J. G. Ma, ${ }^{8}$ Y. G. Ma, ${ }^{38}$ D. Magestro, ${ }^{28}$ S. Mahajan, ${ }^{19}$ D. P. Mahapatra, ${ }^{15}$ R. Majka, ${ }^{49}$ L. K. Mangotra, ${ }^{19}$

R. Manweiler, ${ }^{43}$ S. Margetis, ${ }^{20}$ C. Markert, ${ }^{20}$ L. Martin, ${ }^{39}$ J. N. Marx, ${ }^{21}$ H. S. Matis, ${ }^{21}$ Yu. A. Matulenko, ${ }^{31}$ C. J. McClain, ${ }^{1}$ T. S. McShane, ${ }^{10}$ F. Meissner, ${ }^{21}$ Yu. Melnick, ${ }^{31}$ A. Meschanin, ${ }^{31}$ M. L. Miller, ${ }^{22}$ N. G. Minaev, ${ }^{31}$ C. Mironov, ${ }^{20}$ A. Mischke, ${ }^{27}$ D. K. Mishra, ${ }^{15}$ J. Mitchell, ${ }^{35}$ B. Mohanty, ${ }^{44}$ L. Molnar, ${ }^{32}$ C. F. Moore, ${ }^{41}$ D. A. Morozov, ${ }^{31}$ M. G. Munhoz, ${ }^{36}$ B. K. Nandi, ${ }^{16}$

S. K. Nayak, ${ }^{19}$ T. K. Nayak, ${ }^{44}$ J. M. Nelson, ${ }^{3}$ P. K. Netrakanti, ${ }^{44}$ V. A. Nikitin, ${ }^{13}$ L. V. Nogach, ${ }^{31}$ S. B. Nurushev, ${ }^{31}$

G. Odyniec, ${ }^{21}$ A. Ogawa, ${ }^{4}$ V. Okorokov, ${ }^{25}$ M. Oldenburg, ${ }^{21}$ D. Olson, ${ }^{21}$ S. K. Pal, ${ }^{44}$ Y. Panebratsev, ${ }^{12}$ S. Y. Panitkin, ${ }^{4}$

A. I. Pavlinov, ${ }^{47}$ T. Pawlak, ${ }^{45}$ T. Peitzmann, ${ }^{27}$ V. Perevoztchikov, ${ }^{4}$ C. Perkins, ${ }^{6}$ W. Peryt, ${ }^{45}$ V. A. Petrov, ${ }^{47}$ S. C. Phatak, ${ }^{15}$

R. Picha, ${ }^{7}$ M. Planinic, ${ }^{50}$ J. Pluta, ${ }^{45}$ N. Porile,${ }^{32}$ J. Porter, ${ }^{46}$ A. M. Poskanzer, ${ }^{21}$ M. Potekhin, ${ }^{4}$ E. Potrebenikova, ${ }^{12}$

B. V. K. S. Potukuchi, ${ }^{19}$ D. Prindle, ${ }^{46}$ C. Pruneau, ${ }^{47}$ J. Putschke, ${ }^{21}$ G. Rakness,${ }^{30}$ R. Raniwala, ${ }^{34}$ S. Raniwala, ${ }^{34}$ O. Ravel, ${ }^{39}$ R. L. Ray, ${ }^{41}$ S. V. Razin, ${ }^{12}$ D. Reichhold, ${ }^{32}$ J. G. Reid, ${ }^{46}$ J. Reinnarth, ${ }^{39}$ G. Renault, ${ }^{39}$ F. Retiere, ${ }^{21}$ A. Ridiger, ${ }^{25}$ H. G. Ritter, ${ }^{21}$ J. B. Roberts, ${ }^{35}$ O. V. Rogachevskiy, ${ }^{12}$ J. L. Romero, ${ }^{7}$ A. Rose ${ }^{21}$ C. Roy, ${ }^{39}$ L. Ruan, ${ }^{37}$ M. J. Russcher, ${ }^{27}$ R. Sahoo, ${ }^{15}$

I. Sakrejda, ${ }^{21}$ S. Salur, ${ }^{49}$ J. Sandweiss, ${ }^{49}$ M. Sarsour, ${ }^{40}$ I. Savin, ${ }^{13}$ P. S. Sazhin, ${ }^{12}$ J. Schambach, ${ }^{41}$ R. P. Scharenberg, ${ }^{32}$

N. Schmitz, ${ }^{23}$ K. Schweda, ${ }^{21}$ J. Seger, ${ }^{10}$ I. Selyuzhenkov, ${ }^{47}$ P. Seyboth, ${ }^{23}$ E. Shahaliev, ${ }^{12}$ M. Shao, ${ }^{37}$ W. Shao, ${ }^{5}$ M. Sharma, ${ }^{29}$

W. Q. Shen, ${ }^{38}$ K. E. Shestermanov, ${ }^{31}$ S. S. Shimanskiy, ${ }^{12}$ E. Sichtermann, ${ }^{21}$ F. Simon, ${ }^{22}$ R. N. Singaraju, ${ }^{44}$ N. Smirnov, ${ }^{49}$

R. Snellings, ${ }^{27}$ G. Sood, ${ }^{43}$ P. Sorensen, ${ }^{4}$ J. Sowinski, ${ }^{17}$ J. Speltz, ${ }^{18}$ H. M. Spinka, ${ }^{1}$ B. Srivastava, ${ }^{32}$ A. Stadnik, ${ }^{12}$

T. D. S. Stanislaus, ${ }^{43}$ R. Stock, ${ }^{14}$ A. Stolpovsky, ${ }^{47}$ M. Strikhanov ${ }^{25}$ B. Stringfellow, ${ }^{32}$ A. A. P. Suaide, ${ }^{36}$ E. Sugarbaker, ${ }^{28}$ M. Sumbera, ${ }^{11}$ B. Surrow, ${ }^{22}$ M. Swanger, ${ }^{10}$ T. J. M. Symons, ${ }^{21}$ A. Szanto de Toledo, ${ }^{36}$ A. Tai, ${ }^{8}$ J. Takahashi, ${ }^{36}$ A. H. Tang, ${ }^{27}$ T. Tarnowsky, ${ }^{32}$ D. Thein, ${ }^{8}$ J. H. Thomas, ${ }^{21}$ A. R. Timmins,${ }^{3}$ S. Timoshenko, ${ }^{25}$ M. Tokarev, ${ }^{12}$ T. A. Trainor, ${ }^{46}$ S. Trentalange ${ }^{8}$ R. E. Tribble, ${ }^{40}$ O. D. Tsai, ${ }^{8}$ J. Ulery, ${ }^{32}$ T. Ullrich, ${ }^{4}$ D. G. Underwood, ${ }^{1}$ G. van Buren, ${ }^{4}$ N. van der Kolk, ${ }^{27}$ M. van Leeuwen, ${ }^{21}$ A. M. Vander Molen, ${ }^{24}$ R. Varma, ${ }^{16}$ I. M. Vasilevski, ${ }^{13}$ A. N. Vasiliev, ${ }^{31}$ R. Vernet, ${ }^{18}$ S. E. Vigdor, ${ }^{17}$ Y. P. Viyogi, ${ }^{44}$ S. Vokal, ${ }^{12}$ S. A. Voloshin, ${ }^{47}$ W. T. Waggoner, ${ }^{10}$ F. Wang, ${ }^{32}$ G. Wang, ${ }^{20}$ G. Wang, ${ }^{5}$ X. L. Wang, ${ }^{37}$ Y. Wang, ${ }^{41}$ Y. Wang, ${ }^{42}$ Z. M. Wang, ${ }^{37}$ H. Ward, ${ }^{41}$ J. W. Watson, ${ }^{20}$ J. C. Webb, ${ }^{17}$ G. D. Westfall, ${ }^{24}$ A. Wetzler, ${ }^{21}$ C. Whitten Jr., ${ }^{8}$ H. Wieman, ${ }^{21}$ S. W. Wissink, ${ }^{17}$

R. Witt, ${ }^{2}$ J. Wood, ${ }^{8}$ J. Wu, ${ }^{37}$ N. Xu, ${ }^{21}$ Z. Xu, ${ }^{4}$ Z. Z. Xu, ${ }^{37}$ E. Yamamoto, ${ }^{21}$ P. Yepes,${ }^{35}$ I.-K. Yoo, ${ }^{33}$ V. I. Yurevich, ${ }^{12}$

I. Zborovsky, ${ }^{11}$ H. Zhang, ${ }^{4}$ W. M. Zhang, ${ }^{20}$ Y. Zhang, ${ }^{37}$ Z. P. Zhang, ${ }^{37}$ C. Zhong, ${ }^{38}$ R. Zoulkarneev, ${ }^{13}$ Y. Zoulkarneeva, ${ }^{13}$ A. N. Zubarev, ${ }^{12}$ and J. X. Zuo ${ }^{38}$

(STAR Collaboration)

${ }^{1}$ Argonne National Laboratory, Argonne, Illinois 60439, USA

${ }^{2}$ University of Bern, 3012 Bern, Switzerland

${ }^{3}$ University of Birmingham, Birmingham, United Kingdom

${ }^{4}$ Brookhaven National Laboratory, Upton, New York 11973, USA

${ }^{5}$ California Institute of Technology, Pasadena, California 91125, USA

${ }^{6}$ University of California, Berkeley, California 94720, USA 


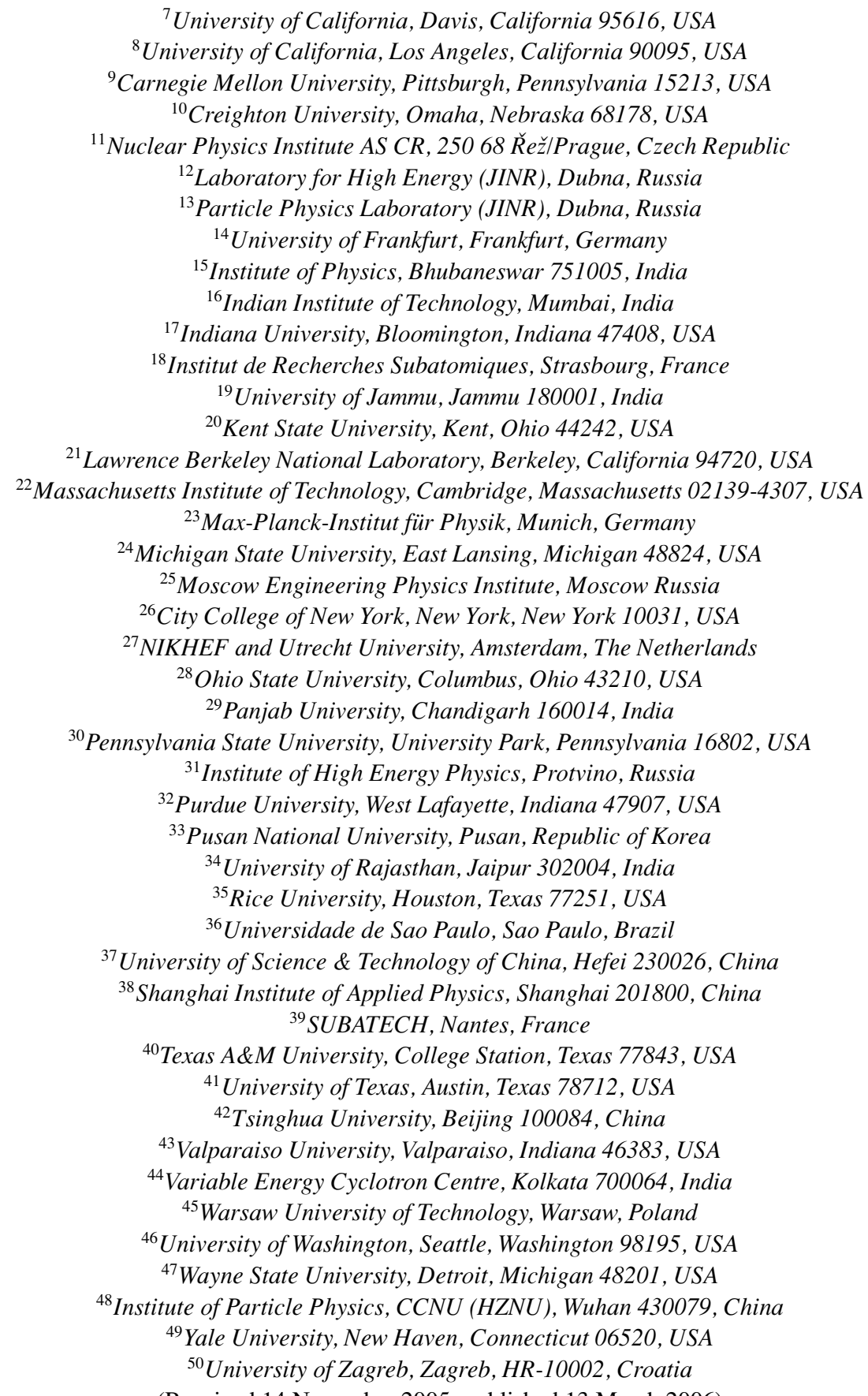

(Received 14 November 2005; published 13 March 2006)

We present the centrality-dependent measurement of multiplicity and pseudorapidity distributions of charged particles and photons in $\mathrm{Au}+\mathrm{Au}$ collisions at $\sqrt{s_{N N}}=62.4 \mathrm{GeV}$. The charged particles and photons are measured in the pseudorapidity region $2.9 \leqslant \eta \leqslant 3.9$ and $2.3 \leqslant \eta \leqslant 3.7$, respectively. We have studied the scaling of particle production with the number of participating nucleons and the number of binary collisions. The photon and charged particle production in the measured pseudorapidity range has been shown to be consistent with energy-independent limiting fragmentation behavior. Photons are observed to follow a centrality-independent limiting fragmentation behavior, while for charged particles it is centrality dependent. We have carried out a comparative study of the pseudorapidity distributions of positively charged hadrons, negatively charged hadrons, photons, pions, and net protons in nucleus-nucleus collisions and pseudorapidity distributions from $p+p$ collisions. From these comparisons, we conclude that baryons in the inclusive charged particle distribution are responsible for the observed centrality dependence of limiting fragmentation. The mesons are found to follow an energy-independent behavior of limiting fragmentation, whereas the behavior of baryons is energy dependent. 


\section{INTRODUCTION}

The STAR experiment [1] at the Relativistic Heavy Ion Collider (RHIC) at Brookhaven National Laboratory has the unique capability of measuring charged particle and photon multiplicities, precisely and simultaneously, at forward rapidity. By using this capability, we can carry out a systematic study of various aspects of charged particle and photon production in relativistic heavy ion collisions.

The conventional way of describing particle production in heavy ion collisions is by measuring the particle density in pseudorapidity $\eta$. Within the framework of certain model assumptions, it provides information on energy density, initial temperature, and velocity of sound in the medium formed in the collisions [2]. The widths of the pseudorapidity distributions are sensitive to longitudinal flow and rescattering effects $[3,4]$. The variation of particle density in $\eta$ with collision centrality, expressed in terms of the number of participating nucleons $N_{\text {part }}$ and/or the number of binary collisions $N_{\text {coll }}$, can shed light on the relative importance of soft versus hard processes in particle production. The particle density in pseudorapidity also provides a test ground for various particle production models, such as those based on ideas of parton saturation [5] and semiclassical QCD, also known as the color glass condensate (CGC) [6].

At RHIC, the particle production mechanism could be different in different regions of pseudorapidity. At midrapidity, a significant increase in charged particle production normalized to the number of participating nucleons has been observed from peripheral to central $\mathrm{Au}+\mathrm{Au}$ collisions [7]. This has been attributed to the onset of hard scattering processes, which scale with the number of binary collisions. However, the total charged particle multiplicity per participant pair, integrated over the whole pseudorapidity range, is independent of centrality in $\mathrm{Au}+\mathrm{Au}$ collisions [8]. In the framework of the color glass condensate picture of particle production [6], the centrality dependence of particle production at midrapidity reflects the increase of gluon density due to the decrease in the effective strong coupling constant. So far, the comparative study of scaling of the photon and the charged particle production with the number of participating nucleons and with the number of binary collisions in a common $\eta$ coverage at forward rapidity has not been reported.

The increase in particle production at midrapidity with increasing center-of-mass energy has been studied in detail at RHIC [8]. It is also of interest to see how particle production varies with center-of-mass energy at forward rapidity. The experimental data on hadron multiplicity and its energy, centrality, and rapidity dependence so far have been consistent with the approach based on ideas of parton saturation. Recently, it has been argued that this onset of saturation occurs somewhere in the center-of-mass energy $\sqrt{s_{N N}}$ range of 17 to $130 \mathrm{GeV}$ [9]. This is one of the reasons cited for having different mechanisms of particle production at RHIC and CERN's super proton synchroton (SPS). The present experimental data at $\sqrt{s_{N N}}=62.4 \mathrm{GeV}$ may help us understand the transition energy for the onset of saturation effects in particle production.

It has been observed that the number of particles (both charged particles and photons) produced per participant pair as a function of $\eta-y_{\text {beam }}$, where $y_{\text {beam }}$ is the beam rapidity, is independent of beam energy $[8,10]$ at forward rapidities. This longitudinal scaling of particle production at forward rapidties is known as limiting fragmentation [11]. In this paper, we extend this definition from the beam energy dependence to the centrality dependence. The inclusive photon production (primarily from decay of $\pi^{0}$ ) at $\sqrt{s_{N N}}=62.4 \mathrm{GeV}$ [10] follows a centrality-independent limiting fragmentation [11] behavior. The inclusive charged particles at 19.6 and $200 \mathrm{GeV}$ have been observed to follow a centrality-dependent behavior of limiting fragmentation [8]. It has been speculated that the baryons, an important constituent of inclusive charged particles, are responsible for the observed difference between photons and charged particles $[8,10]$. The baryons coming from nuclear remnants and baryon transport, both of which change with centrality, may be the source of the centrality-dependent limiting fragmentation for inclusive charged particles. The role of a new mechanism of baryon production as discussed in Refs. [12,13] also needs to be understood. A comparative study of limiting fragmentation of positively and negatively charged particles and photons at the same collision energy and pseudorapidity interval as provided by the present data will help us understand the sources responsible for the observed features. On the theoretical side, reproducing the energy, centrality, and species dependence of limiting fragmentation observed in the experimental data can be a good test for various particle production models. One such attempt to explain the energy dependence of limiting fragmentation phenomena within the framework of CGC has been carried out in Ref. [14]. The importance of the limiting fragmentation curve on energy dependence of particle production has been demonstrated in Ref. [15].

Event-by-event measurements of photon and charged particle multiplicities can be used to study multiplicity fluctuations [16]. Fluctuations in physical observables in heavy ion collisions may provide important information regarding the formation of a quark-gluon plasma and help address the question of thermalization [17]. The study of event-byevent fluctuations in the ratio of photon to charged particle multiplicities has also been proposed as a tool to search for production of disoriented chiral condensates (DCCs) [18].

In this paper, we address some of the above physics issues through the first simultaneous measurement of the charged particle and photon multiplicities for $\mathrm{Au}+\mathrm{Au}$ collisions at $\sqrt{s_{N N}}=62.4 \mathrm{GeV}$ in the forward rapidity. The charged particles are detected using the forward time projection chamber (FTPC), and the photons are detected using the photon multiplicity detector (PMD) in the STAR experiment $[1,19,20]$.

The paper is organized as follows: In Sec. II, we briefly describe the detectors used for measuring the charged particle and photon multiplicities and the trigger detectors used for selecting the minimum bias data, used in the present analysis. In Sec. III, we give details of the data analysis from the FTPC and the PMD. In Sec. IV, we present the results in terms of multiplicity and pseudorapidity distributions of photons and charged particles, scaling of particle production with number of participating nucleons and number of binary collisions, and limiting fragmentation behavior for charged, neutral, and identified particles. Finally we summarize our study in Sec. V. 


\section{DETECTORS}

The STAR experiment [1] consists of several detectors to measure hadronic and electromagnetic observables spanning a large region of the available phase space at RHIC. The detectors used in the present analysis are the FTPC, PMD, a set of trigger detectors used for obtaining the minimum bias data, and the time projection chamber (TPC), data from which are used to determine the collision centrality. The FTPCs, PMD, minimum bias trigger, and collision centrality selection are briefly described below.

\section{A. Forward time projection chambers}

There are two FTPCs; they are located on each side of the nominal collision vertex, around the beam axis. They are cylindrical in structure with a diameter of $75 \mathrm{~cm}$ and $120 \mathrm{~cm}$ in length. Each FTPC has ten rows of readout pads, called padrows, which in turn are subdivided into six sectors with 160 pads per sector. The first padrow is located about $1.63 \mathrm{~m}$ away on both sides from the center of the TPC (the nominal collision point). The sensitive medium is a gas mixture of $\mathrm{Ar}$ and $\mathrm{CO}_{2}$ in the ratio of 1:1 by weight. The FTPCs detect charged particles in the pseudorapidity region $2.5 \leqslant|\eta| \leqslant 4.0$. The novel design of the FTPCs uses a radial drift field, perpendicular to the magnetic field, to achieve a two-track resolution up to $2 \mathrm{~mm}$. This allows for track reconstruction in the environment of high particle density at forward rapidity. In the present analysis, the data from only the FTPC in the positive pseudorapidity region $(2.9 \leqslant \eta \leqslant 3.9)$ is used. Particle production models such as HIJING [21] and AMPT [22] show that about $6-7 \%$ of the total charged particles produced fall within the acceptance of each of the FTPCs. Further details of the design characteristics of the FTPC can be found in Ref. [19].

\section{B. Photon multiplicity detector}

The PMD is located $5.4 \mathrm{~m}$ away from the center of the TPC (the nominal collision point) along the beam axis. It consists of two planes (charged particle veto and preshower) of an array of cellular gas proportional counters. A lead plate of 3 radiation length thickness is placed between the two planes and is used as a photon converter. The sensitive medium is a gas mixture of $\mathrm{Ar}$ and $\mathrm{CO}_{2}$ in the ratio of $7: 3$ by weight. There are 41472 cells in each plane, which are placed inside 12 high-voltage insulated and gastight chambers called supermodules. A photon traversing the converter produces an electromagnetic shower in the preshower plane, leading to a larger signal spread over several cells as compared to a charged particle, which is essentially confined to one cell. The PMD detects photons in the pseudorapidity region $2.3 \leqslant \eta \leqslant 3$.7. The present analysis used only the data from the preshower plane. From HIJING [21] and AMPT [22], we find that about 10-11\% of the total photons produced fall within the acceptance of the PMD. Photon production is dominated by photons from the decay of $\pi^{0} \mathrm{~s}$ [10]. HIJING calculations indicate that about 93-96\% of photons are from inclusive $\pi^{0}$ decays. Further details of the design and characteristics of the PMD can be found in Ref. [20].
TABLE I. Centrality selection, number of participating nucleons, and number of binary collisions.

\begin{tabular}{lccc}
\hline \hline \% cross section & $N_{\text {ch }}^{\mathrm{TPC}}$ & $\left\langle N_{\text {part }}\right\rangle$ & $\left\langle N_{\text {coll }}\right\rangle$ \\
\hline $0-5$ & $>373$ & $347.3_{-3.7}^{+4.3}$ & $904.3_{-62.4}^{+67.7}$ \\
$5-10$ & $373-313$ & $293.3_{-5.6}^{+7.3}$ & $713.7_{-54.8}^{+63.7}$ \\
$10-20$ & $313-222$ & $229.0_{-7.7}^{+9.2}$ & $511.8_{-48.2}^{+54.9}$ \\
$20-30$ & $222-154$ & $162.0_{-9.5}^{+10.0}$ & $320.9_{-39.2}^{+43.0}$ \\
$30-40$ & $154-102$ & $112.0_{-9.1}^{+9.6}$ & $193.5_{-30.4}^{+31.4}$ \\
$40-50$ & $102-65$ & $74.2_{-8.5}^{+9.0}$ & $109.3_{-21.8}^{+22.1}$ \\
$50-60$ & $65-38$ & $45.8_{-7.1}^{+7.0}$ & $56.6_{-14.3}^{+155.0}$ \\
$60-70$ & $38-20$ & $25.9_{-5.6}^{+5.6}$ & $26.8_{-9.0}^{+8.8}$ \\
$70-80$ & $20-9$ & $13.0_{-4.6}^{+3.4}$ & $11.2_{-4.8}^{+3.7}$ \\
\hline \hline
\end{tabular}

\section{Minimum bias trigger and collision centrality}

The minimum bias trigger is obtained using the charged particle hits from an array of scintillator slats arranged in a barrel, called the central trigger barrel, surrounding the TPC, two zero-degree hadronic calorimeters at $\pm 18 \mathrm{~m}$ from the detector center along the beam line, and two beam-beam counters [23]. The centrality determination in this analysis uses the uncorrected multiplicity of charged particles in the pseudorapidity region $|\eta|<0.5$, as measured by the TPC [24]. Table I gives the percentage cross section, the corresponding uncorrected multiplicity of charged particle tracks $N_{\mathrm{ch}}^{\mathrm{TPC}}$ in the pseudorapidity region $|\eta|<0.5$, the number of participating nucleons $N_{\text {part }}$, and the number of binary collisions $N_{\text {coll }}$ used in this paper. The number of participating nucleons and the number of binary collisions have been obtained from Monte Carlo Glauber calculations [24] using the Woods-Saxon distribution for the nucleons inside the gold nucleus. The systematic uncertainties on $N_{\text {part }}$ and $N_{\text {coll }}$ were determined by varying the Woods-Saxon parameters and by including a $5 \%$ uncertainty in the determination of the total measured $\mathrm{Au}+\mathrm{Au}$ cross section. The contributions from these sources were determined seperately and treated as fully correlated in the final systematic uncertainties presented in the Table I.

\section{DATA RECONSTRUCTION}

\section{A. Charged particle reconstruction}

The analysis of the data from the FTPC involves the following steps: (a) event selection, (b) pad-to-pad gain calibration, and (c) reconstruction of charged tracks.

A total of 1.2 million minimum bias events, corresponding to $0-80 \%$ of the $\mathrm{Au}+\mathrm{Au}$ hadronic interaction cross section, have been selected with a collision vertex position less than $30 \mathrm{~cm}$ from the center of the TPC along the beam axis.

The calibration of the FTPC is done using a laser calibration system [19]. This system helps to calibrate the drift velocity in the nonuniform radial drift field and also provides information for making corrections to spatial distortions caused by mechanical or drift field imperfections. The localization of dead pads is done with pulsers and by an analysis of data to identify electronically noisy pads [25]. 
The reconstruction of experimental data involves two steps: (a) cluster-finding to calculate the track points from the charge distribution detected by the pads and (b) track-finding to group the track points of different padrows of the FTPC to form a track. Cluster-finding includes reading the electronic signal data from the data acquisition system, looking for areas of nonzero charge (cluster), deconvoluting the clusters, and then finding the point coordinates. This is followed by combining clusters from all padrows to form tracks using a suitable tracking algorithm [26]. A track is considered valid if it consists of at least five found clusters and if its distance of closest approach to the primary vertex is less than $3 \mathrm{~cm}$. The condition of having at least five found clusters for each track in the FTPC ensures a small contribution of split tracks. The split tracks contribution and background contamination are primarily from $\gamma$ conversion electrons and positrons which are significantly reduced when we include those tracks in the analysis, which have transverse momentum in the range $0.1<$ $p_{\mathrm{T}}<3 \mathrm{GeV} / c$. The maximum percentage of split tracks was estimated from simulations to be $\sim 1.5 \%$. Two procedures are used to obtain the charged particle yields at all $p_{\mathrm{T}}$. The charged particle transverse momentum spectra are fitted by a power-law function in the range $0.1<p_{\mathrm{T}}<1 \mathrm{GeV} / c$ and extrapolated to $p_{\mathrm{T}}=0 \mathrm{GeV} / c$. The low $p_{\mathrm{T}}$ yield is obtained from this extrapolation. The other procedure calculates the yield of charged particles for $p_{\mathrm{T}}<0.1 \mathrm{GeV} / c$ by using the ratio of the yield in this $p_{\mathrm{T}}$ range to total yields from HIJING [21] simulations. Both these procedures resulted in correction factors of the order of $14 \%$ and $16 \%$, respectively, in the region $2.9 \leqslant \eta \leqslant 3.9$.

The efficiency of charged particle reconstruction $\epsilon_{\mathrm{ch}}$ as a function of pseudorapidity is estimated by embedding Monte Carlo charged tracks into real data and then following the full reconstruction chain [27]. The reconstruction efficiency is obtained by dividing the number of reconstructed Monte Carlo tracks within an $\eta$ bin by the total number of embedded Monte Carlo tracks in the same $\eta$ bin. The charged particle reconstruction efficiencies for central and peripheral collisions, for the $\eta$ region studied, are shown in Fig. 1. The background

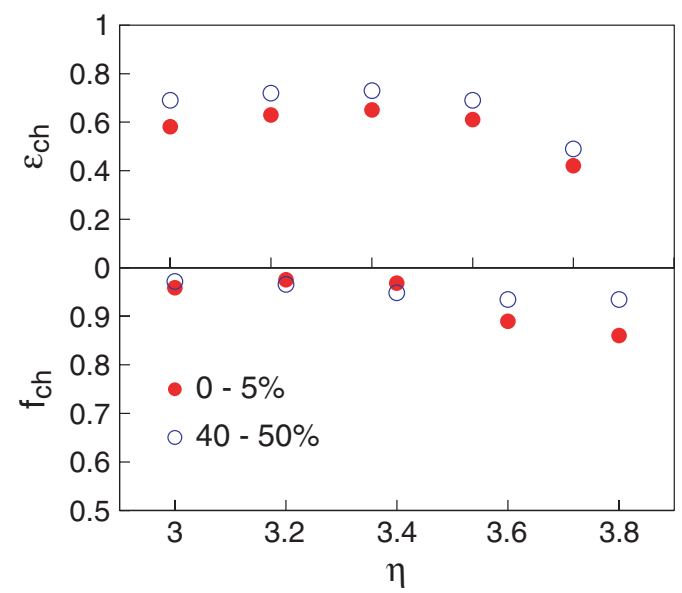

FIG. 1. (Color online) Charged particle reconstruction efficiency $\epsilon_{\mathrm{ch}}$ and purity of charged hadron sample $f_{\mathrm{ch}}$ in the FTPC as a function of pseudorapidity $\eta$ for charged tracks with $0.1<p_{\mathrm{T}}<3 \mathrm{GeV} / c$, for two centrality intervals. contamination is obtained from detailed Monte Carlo simulation using the HIJING (version 1.382) event generator [21] and the detector simulation package GEANT [28], which incorporates the full STAR detector framework. The purity of the charged hadron sample $f_{\text {ch }}$ in the FTPC for central and peripheral collisions is also shown in Fig. 1. The errors on efficiency and purity values will be discussed later.

\section{B. Photon reconstruction}

Data analysis from the PMD involves: (a) event selection, (b) cell-to-cell gain calibration, and (c) reconstruction or extraction of photon multiplicity.

A total of 0.3 million minimum bias events, corresponding to $0-80 \%$ of the $\mathrm{Au}+\mathrm{Au}$ hadronic interaction cross section, were selected with a collision vertex position less than $30 \mathrm{~cm}$ from the center of the TPC along the beam axis. The difference in the number of events for the PMD and FTPC analysis originated from the fact that for the same period of data-taking, the PMD recorded fewer events and there was a need for a more stringent data cleanup procedure to remove events with pileup effects.

The cell-to-cell gain calibration was done by obtaining the analog-to-digital conversion (ADC) distributions of isolated cells. Such distribution may be treated as the response of the cell to charged particles [20]. For most of the cells, this response follows a Landau distribution. We used the mean of the ADC distribution of isolated cells to estimate and correct the relative gains of all cells within each supermodule. The cell-to-cell gain variation was $10-25 \%$ for the different supermodules.

The extraction of photon multiplicity proceeds in two steps involving clustering of hits and photon-hadron discrimination. Hit clusters consist of contiguous cell signals. Photons are separated from charged particles using the following conditions: (a) the number of cells in a cluster is $>1$ and (b) the cluster signal is larger than three times the average response of all isolated cells in a supermodule. The choice of the conditions is based on a detailed study of simulations $[10,20]$. The number

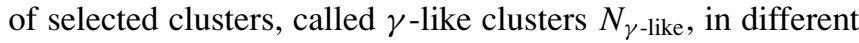
supermodules for the same $\eta$ coverage are used to evaluate the effect of possible nonuniformity in the response of the detector.

To estimate the number of photons $N_{\gamma}$ from the detected $N_{\gamma \text {-like }}$ clusters, we evaluate the photon reconstruction efficiency $\epsilon_{\gamma}$ and purity $f_{p}$ of the $\gamma$-like sample defined [29] as $\epsilon_{\gamma}=N_{\mathrm{cls}}^{\gamma, \text { th }} / N_{\gamma}$ and $f_{p}=N_{\mathrm{cls}}^{\gamma \text {,th }} / N_{\gamma \text {-like }}$, respectively. $N_{\mathrm{cls}}^{\gamma \text {,th }}$ is the number of photon clusters above the photon-hadron discrimination condition. Both $\epsilon_{\gamma}$ and $f_{p}$, are obtained from a detailed Monte Carlo simulation using HIJING [21] with default parameter settings and the detector simulation package GEANT [28], which incorporates the full STAR detector framework. Both $\epsilon_{\gamma}$ and $f_{p}$ vary with pseudorapidity and centrality. This is due to variations in particle density, upstream conversions, and detector-related effects. A photon should ideally create one cluster in the detector. However, it may give rise to more than one cluster (called split clusters) in the real experimental environment. These may happen because of 


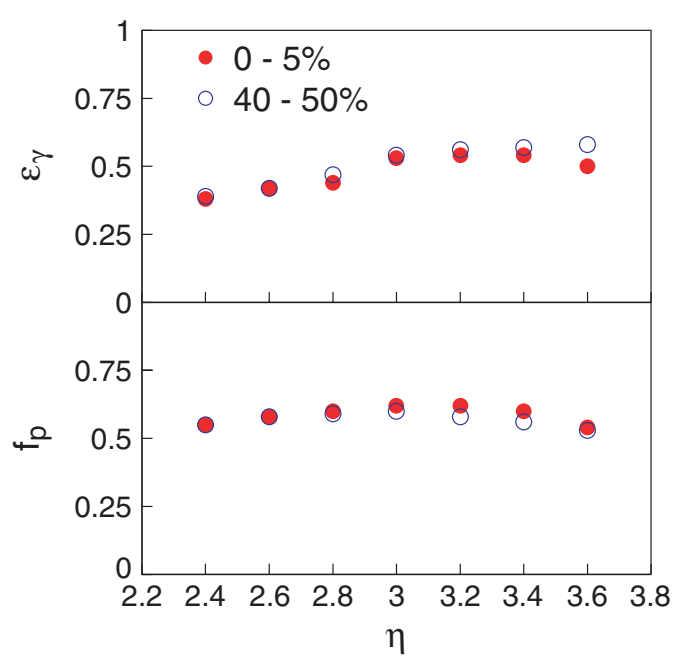

FIG. 2. (Color online) Photon reconstruction efficiency $\epsilon_{\gamma}$ and purity of photon sample $f_{p}$ for PMD as a function of pseudorapidity $\eta$, for two centrality intervals.

conversions of the photon due to upstream materials in front of the PMD, or limitations of the clustering algorithm due to varying particle density. The highest occupancy of the PMD is about $12 \%$, and the maximum percentage of split clusters is estimated to be $9 \%$. The photon reconstruction efficiency and the purity of the photon sample determined by means of simulations for central and peripheral collisions for the $\eta$ region studied are shown in Fig. 2. The lower limit of photon $p_{\mathrm{T}}$ acceptance in the PMD is estimated from detector simulations to be $20 \mathrm{MeV} / c$.

\section{Systematic errors}

The systematic errors on the charged particle multiplicity $N_{\text {ch }}$ are due to uncertainties in estimates of $\epsilon_{\text {ch }}$ and $f_{\text {ch. }}$. The uncertainty in the estimates are obtained through simulations by varying the track quality cuts. The value of the maximal distance of closest approach of a track to the primary vertex is varied by $0.5 \mathrm{~cm}$ leading to a maximum error on $N_{\mathrm{ch}}$ of $\sim 6 \%$. The minimum number of clusters to form a track was varied from five to four. This led to an error in $N_{\mathrm{ch}}$ of $\sim 1 \%$. The uncertainty in the correction factor to obtain the $N_{\mathrm{ch}}$ yield for $p_{\mathrm{T}}<0.1 \mathrm{GeV} / c$ is $\sim 8 \%$. This also contributes to the total systematic errors. The total systematic error in $N_{\mathrm{ch}}$ is $\sim 10 \%$ for all the centrality classes studied. The systematic error for the region $\eta>3.6$ is estimated to be about $15 \%$, due to larger uncertainty in the reconstruction efficiency. This arises primarily because of uncertainty in realistic reproduction of electronic loss, at the extreme ends of the detector acceptance. This is estimated by studying the azimuthal dependence of charged particle density in a given $\eta$ window.

The systematic errors in the photon multiplicity $N_{\gamma}$ are due to (a) uncertainty in estimates of $\epsilon_{\gamma}$ and $f_{p}$ values arising from splitting of clusters and the choice of photon-hadron discrimination threshold and (b) uncertainty in $N_{\gamma}$ arising from the nonuniformity of the detector response primarily due to cell-to-cell gain variation. The error in $N_{\gamma}$ due to (a) is estimated from Monte Carlo simulations to be $9.8 \%$ and $7.7 \%$ in central and peripheral collisions, respectively. The error in $N_{\gamma}$ due to (b) is estimated using average gains for normalization and by studying the azimuthal dependence of the photon density of the detector in an $\eta$ window to be $13.5 \%$ for central and 15\% for peripheral collisions. The total systematic error in $N_{\gamma}$ is $\sim 17 \%$ for both central and peripheral collisions.

The total errors in $N_{\mathrm{ch}}$ and $N_{\gamma}$ are obtained by adding respective systematic and statistical errors in quadrature and are shown in all the figures unless mentioned otherwise. The statistical errors are small and within the symbol sizes.

\section{RESULTS AND DISCUSSION}

\section{A. Multiplicity distributions}

The charged particle multiplicity $N_{\mathrm{ch}}$ and photon multiplicity $N_{\gamma}$ are obtained event-by-event in the FTPC and the PMD following the analysis procedure described above. Figure 3 shows the minimum bias distributions of $N_{\mathrm{ch}}$ and $N_{\gamma}$ for $\mathrm{Au}+\mathrm{Au}$ collisions at $\sqrt{s_{N N}}=62.4 \mathrm{GeV}$. The distributions
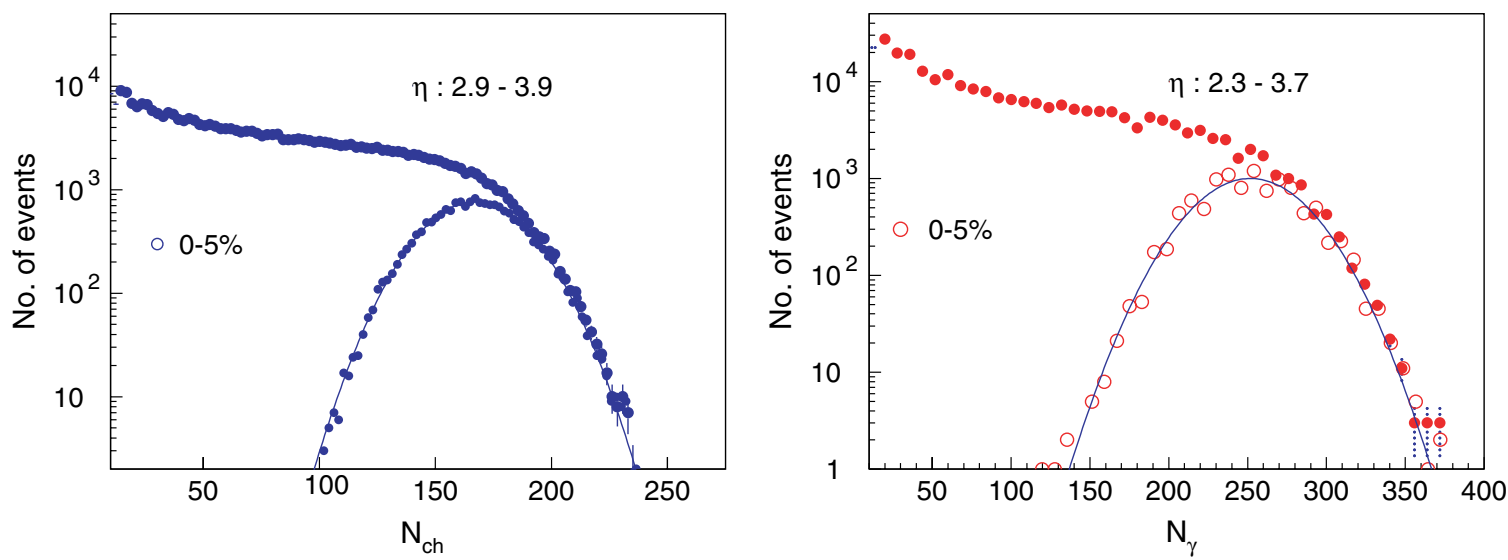

FIG. 3. (Color online) Minimum bias $N_{\mathrm{ch}}(2.9 \leqslant \eta \leqslant 3.9)$ and $N_{\gamma}(2.3 \leqslant \eta \leqslant 3.7)$ distributions for $\mathrm{Au}+\mathrm{Au}$ collisions at $\sqrt{s_{N N}}=62.4 \mathrm{GeV}$. The charged particle and photon multiplicity distribution for top 5\% central events are shown in open circles. Solid curve is the Gaussian fit to the data points. Systematic errors in $N_{\text {ch }}$ and $N_{\gamma}$ are not shown on the data points. 


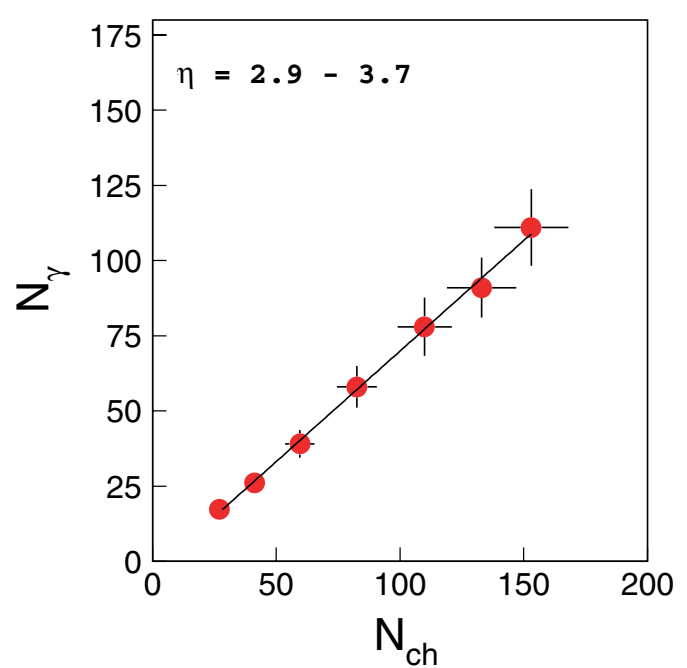

FIG. 4. (Color online) Correlation between average number of charged particles $N_{\text {ch }}$ and average number of photons $N_{\gamma}$ within the common $\eta$ range of FTPC and PMD, $2.9 \leqslant \eta \leqslant 3.7$, for different collision centrality classes in $\mathrm{Au}+\mathrm{Au}$ collisions at $\sqrt{s_{N N}}=62.4 \mathrm{GeV}$. Solid line is a straight line fit to the data points.

have a characteristic shape with a steep rise that corresponds to the most peripheral events. The plateaus in the photon and charged particle multiplicity distributions correspond to midcentral events, and the falloff to the most central collision events. The shape of the curves in the falloff region reflects the intrinsic fluctuations of the measured quantities and the limited acceptance of the detectors. The event-by-event charged particle and photon multiplicity distributions for $0-5 \%$ central collisions are also shown. Gaussian fits to these distributions have been made. The values of the fit parameters for charged particles measured in $2.9 \leqslant \eta \leqslant 3.9$ are a mean of 167 and $\sigma=20$; $\chi^{2} / n d f=70.67 / 69$. The values of the fit parameters for photons measured in $2.3 \leqslant \eta \leqslant 3.7$ are a mean of 252 and $\sigma=30 ; \chi^{2} / n d f=37.3 / 34$. The correlation between the average number of charged particles and average number of photons within the common pseudorapidity coverage of the FTPC and PMD $(2.9 \leqslant \eta \leqslant 3.7)$ for different collision centrality classes in $\mathrm{Au}+\mathrm{Au}$ collisions at $\sqrt{s_{N N}}=62.4 \mathrm{GeV}$ are shown in Fig. 4. The correlation between $N_{\text {ch }}$ and $N_{\gamma}$ can be expressed as $N_{\gamma}=(0.74 \pm 0.01) N_{\text {ch }}-(3.57 \pm 0.83)$. The correlated error on the slope parameter is approximately $12 \%$. This is shown as a straight line in the figure. The correlation reflects the variation of $N_{\gamma}$ and $N_{\text {ch }}$ with collision centrality. The correlation coefficient is $1.01 \pm 0.01$.

\section{B. Scaling of particle production}

After having discussed the event-by-event measurement of photon and charged particle multiplicities in the previous section, we now discuss the variation of average (averaged over number of events) photon and charged particle multiplicities within the full coverage of the PMD and FTPC, respectively, with centrality. Collision centrality is expressed in terms of either number of participating nucleons or number of binary collisions. This will provide information on the contribution

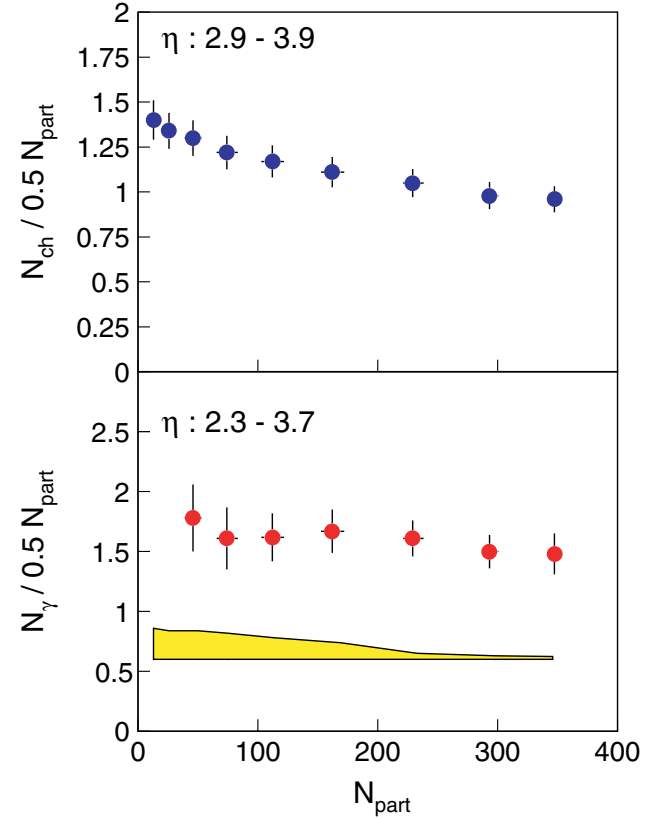

FIG. 5. (Color online) Variation of $N_{\mathrm{ch}}$ normalized to the number of participating nucleon pair in the FTPC coverage $(2.9 \leqslant \eta \leqslant 3.9)$ and $N_{\gamma}$ normalized to the number of participating nucleon pair in the PMD acceptance $(2.3 \leqslant \eta \leqslant 3.7)$ as a function of $N_{\text {part }}$. Lower band shows uncertainty in the ratio due to uncertainties in $N_{\text {part }}$ calculations.

of hard (pQCD jets) and soft processes to particle production at forward rapidity. The scaling of particle production with the number of participating nucleons indicates the dominance of soft processes, whereas scaling with the number of binary collisions indicates the onset of hard processes. At midrapidity, the particle production at $\sqrt{s_{N N}}=130$ and $200 \mathrm{GeV}$ has been shown to scale with a combination of $N_{\text {part }}$ and $N_{\text {coll }}$ [7]. Here, we present the results on scaling of particle production at forward rapidity for $\mathrm{Au}+\mathrm{Au}$ collisions at $\sqrt{s_{N N}}=62.4 \mathrm{GeV}$.

Figure 5 shows the variation of the total number of charged particles in the FTPC coverage $(2.9 \leqslant \eta \leqslant 3.9)$ and the total number of photons in the PMD acceptance $(2.3 \leqslant \eta \leqslant 3.7)$, both normalized to $N_{\text {part }}$, as a function of the collision centrality, expressed by the number of participants. Higher $N_{\text {part }}$ values correspond to more central collisions or collisions with smaller impact parameter. The charged particle yield per participating nucleon pair at forward rapidity decreases from peripheral to central collisions. The photon production per participant pair is found to be approximately constant with centrality in the forward $\eta$ range studied.

Figure 6 shows the variation of the total number of charged particles normalized to the number of collisions in the FTPC coverage $(2.9 \leqslant \eta \leqslant 3.9)$ and the total number of photons normalized to the number of collisions in the PMD coverage $(2.3 \leqslant \eta \leqslant 3.7)$ as a function of the number of binary collisions. Higher $N_{\text {coll }}$ values correspond to more central collisions or collisions with smaller impact parameter. Both the charged particle yield and photon yield normalized to the number of binary collisions do not scale with the number of binary collisions at forward rapidity. The data value decreases from peripheral to central collisions. This indicates that the 


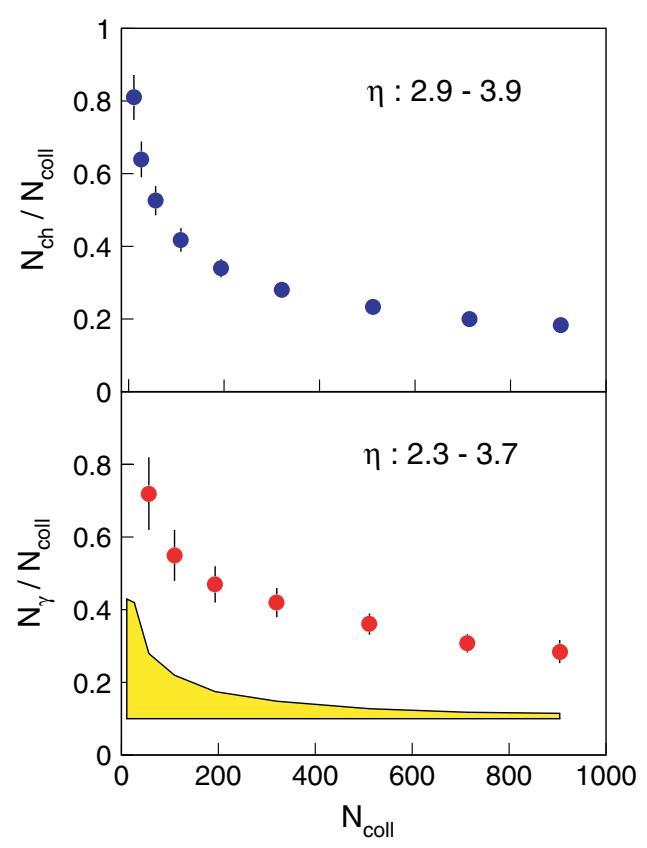

FIG. 6. (Color online) Same as Fig. 5, but normalized to the number of collisions in the FTPC and PMD coverages as a function of $N_{\text {coll }}$.

contribution of hard processes to particle production at forward rapidity is small. Due to the large uncertainty associated with photon reconstruction, data for lower centrality bins are not presented.

\section{Pseudorapidity distributions}

In this section, we study the variation in particle density with $\eta$. The results can then be directly compared to different models in order to understand the mechanism of particle production in heavy ion collisions at forward rapidity.

Figure 7 shows the pseudorapidity distributions of charged particles within $2.9 \leqslant \eta \leqslant 3.9$ and photons within $2.3 \leqslant \eta \leqslant 3.7$ for various event centrality classes. As expected, the particle density increases with decrease in $\eta$. Figure 8 compares of pseudorapidity distributions for photons and charged particles for $0-5 \%$ and $40-50 \%$ central $\mathrm{Au}+\mathrm{Au}$ collisions at $\sqrt{s_{N N}}=$ $62.4 \mathrm{GeV}$ with the corresponding results from various theoretical models. There is an additional 5\% systematic errors because of uncertainty in centrality selection and is not shown in the figure. The HIJING model [21] is based on perturbative QCD processes which lead to multiple jet production and jet interactions in matter. HIJING seems to underpredict the measured photon multiplicity. However, within the systematic errors, it is difficult to make definitive conclusions. For charged particles, HIJING reasonably explains the $\eta$ distributions for central and peripheral collisions at higher $\eta$. The AMPT model [22] is a multiphase transport model which includes both initial partonic and final hadronic interactions. For photons, the results from the AMPT model are in reasonable agreement with the data for central and peripheral events within the systematic errors. For charged particles in central collisions, the results
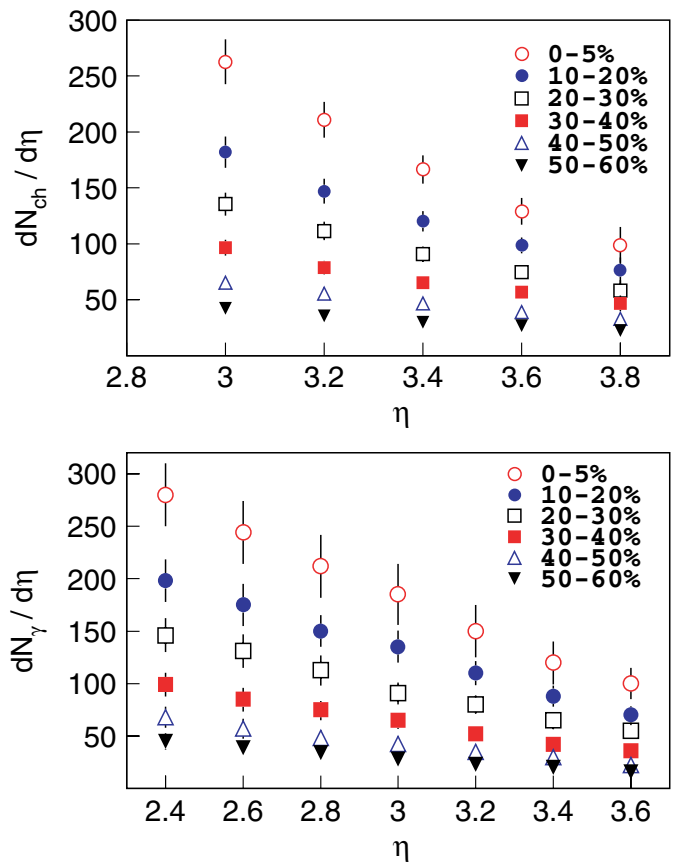

FIG. 7. (Color online) $d N / d \eta$ for charged particles and photons for $\mathrm{Au}+\mathrm{Au}$ collisions at $\sqrt{s_{N N}}=62.4 \mathrm{GeV}$ for various event centrality classes.

from AMPT explain the data at lower $\eta$ and overpredict the charged particle yields at higher $\eta$. The LEXUS model [30] is based on linear extrapolation of nucleon-nucleon collisions to high-energy nucleus-nucleus collisions. For charged particles, the LEXUS model agrees with experimental data at higher $\eta$
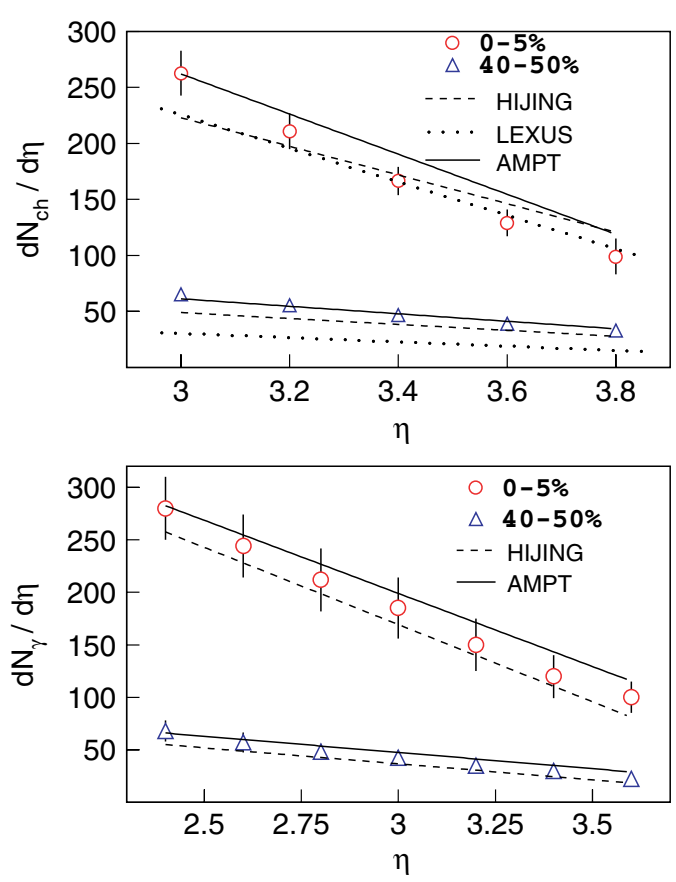

FIG. 8. (Color online) $d N / d \eta$ for charged particles and photons for central and peripheral $\mathrm{Au}+\mathrm{Au}$ collisions at $\sqrt{s_{N N}}=62.4 \mathrm{GeV}$ compared to corresponding results from theoretical models. 
TABLE II. Parameters $C, \eta_{0}$, and $\delta$ for different $\sqrt{s_{N N}}$.

\begin{tabular}{lccr}
\hline \hline$\sqrt{s_{N N}}(\mathrm{GeV})$ & \multicolumn{1}{c}{$\eta_{0}$} & \multicolumn{1}{c}{$\delta$} \\
\hline 19.6 & $382 \pm 33$ & $2.16 \pm 0.17$ & $0.7 \pm 0.06$ \\
$62.4^{\mathrm{a}}$ & $458 \pm 40$ & $3.08 \pm 0.35$ & $0.69 \pm 0.06$ \\
130 & $580 \pm 21$ & $3.59 \pm 0.076$ & $0.66 \pm 0.05$ \\
200 & $667 \pm 22$ & $3.80 \pm 0.082$ & $0.71 \pm 0.06$ \\
\hline \hline
\end{tabular}

${ }^{\text {a Interpolated. }}$

for central collisions. It underpredicts the charged particle yields for peripheral collisions. In summary, we observe that the photon and charged particle multiplicity within the systematic errors is reasonably well explained by HIJING and AMPT models.

Now we will (a) discuss the energy dependence of the shape of the $\eta$ distribution of charged particles available at various energies of $\mathrm{Au}+\mathrm{Au}$ collisions in $\mathrm{RHIC}$ for central collisions and (b) try to estimate the full $\eta$ distribution for charged particles for $\sqrt{s_{N N}}=62.4 \mathrm{GeV}$ from the above study and compare our result to the present measurements.

The full pseudorapidity distribution of charged particles at RHIC for central collisions can be parametrized by the following three-parameter formula:

$$
\frac{d N}{d \eta}=\frac{C}{1+\exp \frac{\eta-\eta_{0}}{\delta}} .
$$

This formula is chosen to describe the central plateau and the falloff in the fragmentation region of the distribution by means of the parameters $\eta_{0}$ and $\delta$, respectively. Using this formula, we can describe the 200,130 , and $19.6 \mathrm{GeV}$ pseudorapidity distributions of charged particles from the PHOBOS experiment [8]. The values of the parameters $C, \eta_{0}$, and $\delta$ are given in Table II and the fits to data are shown in Fig. 9. The value of $\eta_{0}$ is found to increase with increasing $\sqrt{s_{N N}}$. The value of the parameter $\delta$ is found to be independent

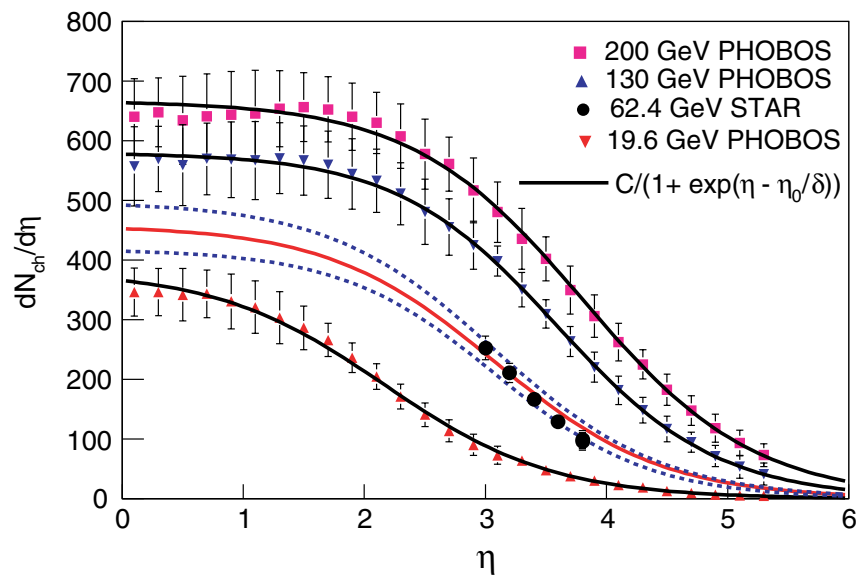

FIG. 9. (Color online) Pseudorapidity distributions of charged particles for various c.m. energies in $\mathrm{Au}+\mathrm{Au}$ central collisions. Pseudorapidity distributions for $0-6 \%$ central $\mathrm{Au}+\mathrm{Au}$ collisions at $\sqrt{s_{N N}}=200,130$, and $19.6 \mathrm{GeV}$ are from the PHOBOS experiment [8]. Solid lines are the results of the fits described in the text.
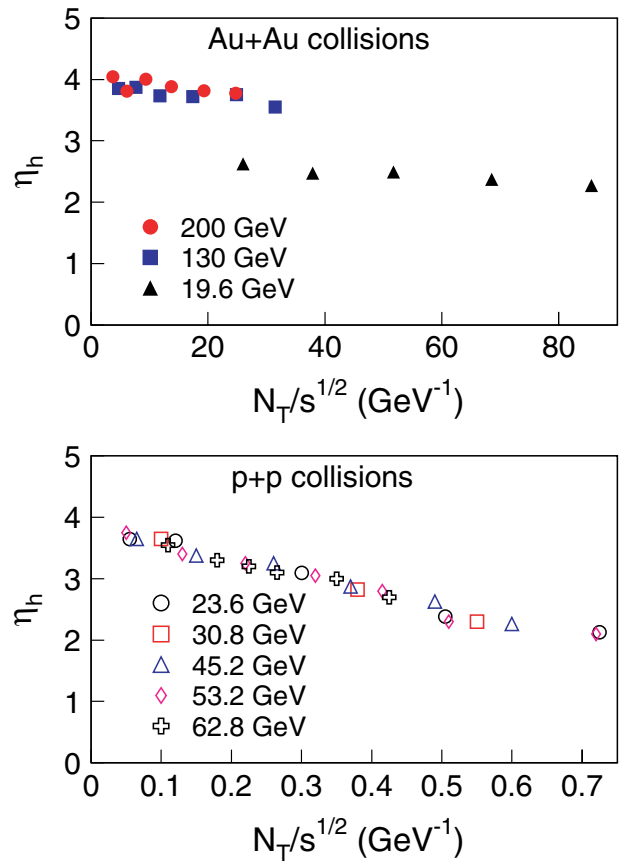

FIG. 10. (Color online) $\eta_{h}$ of charged particles as a function of $N_{T}$ normalized to the center of mass energy. The $\mathrm{Au}+\mathrm{Au}$ collision data are from the PHOBOS [8] experiment, and $p+p$ collision data are from the ISR [31] experiments.

of energy within errors. This feature is another way of testing the concept of limiting fragmentation, which will be discussed later. By using the average value of $\delta$ and interpolating the value of $\eta_{0}$ to $62.4 \mathrm{GeV}$, we are able to predict the full pseudorapidity distribution for charged particles at $62.4 \mathrm{GeV}$. This is shown as the solid curve in Fig. 9, together with our measured charged particle data for $62.4 \mathrm{GeV}$ at forward rapidity. The dashed curves represent the error in obtaining the full pseudorapidity distribution for charged particles using the interpolation method described.

We have also studied the widths of the pseudorapidity distributions of charged particles at RHIC and compared them to those from $p+p$ collisions at Intersecting Storage Rings (ISR) [31]. In Fig. 10, we show the variation of the half width at half maximum $\eta_{h}$ of the charged particle pseudorapidity distributions as a function of total charged particle multiplicity normalized to the center of mass energy $\left(N_{T} / \sqrt{s_{N N}}\right)$ for $p+p$ and $\mathrm{Au}+\mathrm{Au}$ collisions. Data are shown for various centrality classes in $\mathrm{Au}+\mathrm{Au}$ collisions [8] and various intervals of observed total multiplicity in $p+p$ collisions. We observe that the half width at half maximum obeys an interesting scaling law in $p+p$ collisions, and is found to depend on a single variable $\left(N_{T} / \sqrt{s_{N N}}\right)$. In $\mathrm{Au}+\mathrm{Au}$ Collisions, this scaling seems to be valid for 200 and $130 \mathrm{GeV}$. Although the width decreases with $N_{T} / \sqrt{s_{N N}}$ for $19.6 \mathrm{GeV}$, the data lie below the higher energy data unlike the energy-independent behavior observed in $p+p$ collisions. This may reflect the change in the mechanism of particle production over the full pseudorapidity range as we increase the $\sqrt{s_{N N}}$ from 19.6 to $\sqrt{s_{N N}}>130 \mathrm{GeV}$ in $\mathrm{Au}+\mathrm{Au}$ collisions at RHIC. 


\section{Energy dependence of particle multiplicity}

The energy dependence of charged particle yields at midrapidity has been studied at RHIC [8]. Here, we present the results of the energy dependence of particle yields at forward rapidity and compare them with yields at midrapidity.

Figure 11 shows the charged particle pseudorapidity distribution scaled by the number of participating nucleon pairs at midrapidity $(\eta=0)$ and forward rapidity $(\eta=3.0)$ as a function of $\sqrt{s_{N N}}$ for central collisions at RHIC. The data for charged particles at $\sqrt{s_{N N}}=19.6,56,130$, and $200 \mathrm{GeV}$ at $\eta=3.0$ are from the PHOBOS [8] and BRAHMS [32] experiments. The data for charged particles at midrapidity are the averages of the values from the four RHIC experiments. The charged particle production at $\eta=0$ can be expressed as

$$
\begin{aligned}
\frac{d N / d \eta}{0.5 N_{\text {part }}}= & 1.75( \pm 0.25)+0.017( \pm 0.005) \ln \left[\sqrt{s_{N N}}\right] \\
& -0.00003( \pm 0.00002)\left(\ln \left[\sqrt{s_{N N}}\right]\right)^{2}
\end{aligned}
$$

The charged particle production at $\eta=3.0$, can be expressed as

$$
\begin{aligned}
\frac{d N / d \eta}{0.5 N_{\text {part }}}= & -0.03( \pm 0.13)+0.028( \pm 0.004) \ln \left[\sqrt{s_{N N}}\right] \\
& -0.00007( \pm 0.00002)\left(\ln \left[\sqrt{s_{N N}}\right]\right)^{2}
\end{aligned}
$$

The ratio of charged particle production at $\eta=0$ to that at $\eta=3.0$ decreases from a factor of 4 to 1.3 as $\sqrt{s_{N N}}$ increases from 19.6 to $200 \mathrm{GeV}$. The photon result at $\sqrt{s_{N N}}=62.4 \mathrm{GeV}$ for $\eta=3.0$ is also shown. The photon yields at other $\sqrt{s_{N N}}$ values at forward rapidity and midrapidity are not yet available at RHIC. The photon production at $\sqrt{s_{N N}}=62.4 \mathrm{GeV}$ is about $35 \%$ lower than the charged particle production for the same energy at $\eta=3.0$. The charged particle yield at $\eta=3.0$ for

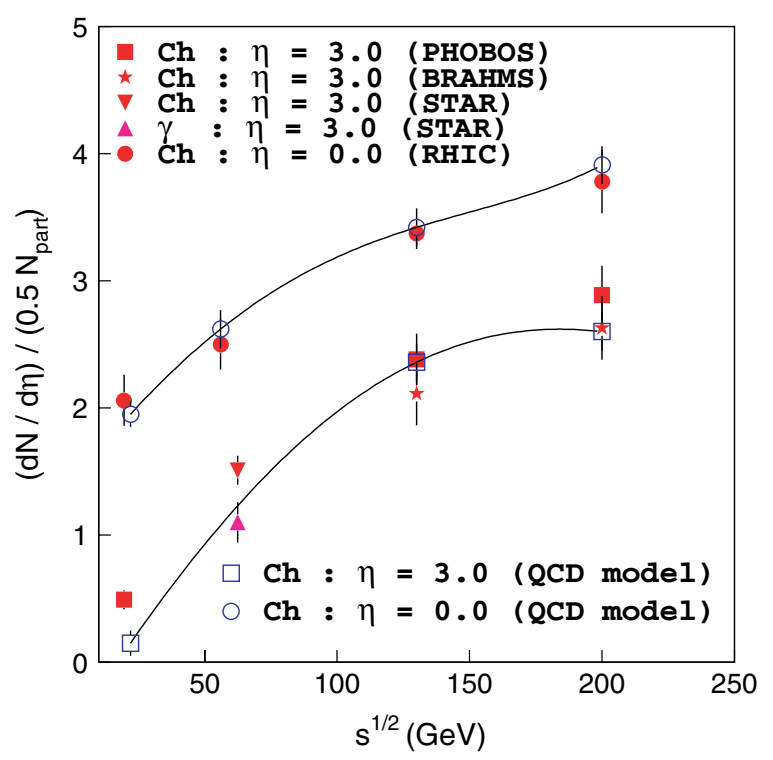

FIG. 11. (Color online) $d N / d \eta$ per participating nucleon pair at midrapidity $(\eta=0)$ and forward rapidity $(\eta=3.0)$ for various c.m. energies for central collisions. Solid lines are polynomial fits to the values from the QCD model, no prediction for $\sqrt{s_{N N}}=62.4 \mathrm{GeV}$ is available from this model.
$\sqrt{s_{N N}}=62.4 \mathrm{GeV}$ is a factor of 1.6 and 1.9 lower than the corresponding yields at 130 and $200 \mathrm{GeV}$ and a factor of 3.0 higher than the charged particle yields at $19.6 \mathrm{GeV}$. For comparison, also shown in Fig. 11 are the results from a model based on parton saturation, which is expected in high-density QCD [33]. The results from the model agree with the measured charged particle yields at midrapidity for all energies at RHIC. However, the model's prediction for forward rapidity at the lowest energy $(22 \mathrm{GeV})$ is lower than the data $(19.6 \mathrm{GeV})$. There is no prediction for $\sqrt{s_{N N}}=62.4 \mathrm{GeV}$ available from this model. It would be interesting to have the predictions to understand the transition energy for the onset of saturation effects at RHIC.

\section{E. Comparison of $N_{\mathrm{ch}}$ and $N_{\gamma}$}

The STAR experiment at RHIC has the unique capability to study the yields of charged particle and photons at forward rapidity. Figure 12 shows the ratio of $N_{\text {ch }}$ to $N_{\gamma}$ for $0-5 \%$ and $40-50 \%$ central $\mathrm{Au}+\mathrm{Au}$ collisions at $\sqrt{s_{N N}}=62.4 \mathrm{GeV}$ as a function of $\eta$ in the common $\eta$ coverage of the FTPC and the PMD. The ratio is around 1.4 for central collisions and 1.6 for peripheral collisions within $3.0<\eta<3.6$. The results from HIJING indicate similar values. The correlated systematic errors ( $\sim 7 \%$ ), mainly arising due to uncertainties in the Monte Carlo determination of reconstruction efficiencies and normalization errors, are not plotted on the data points and are shown as a shaded band. The photon production is dominated by photons from the decay of $\pi^{0} \mathrm{~s}$ [10]. The charged particle yields have a substantial contribution from baryons at forward rapidity [34]. Apart from the kinematics, this may be the reason for higher charged particle yields compared to photons. In the future, event-by-event study of $N_{\mathrm{ch}}$ and $N_{\gamma}$ correlations in common $\eta$ and $\phi$ coverage of the FTPC and the PMD can be used to look for possible formation of disoriented chiral condensates [18].

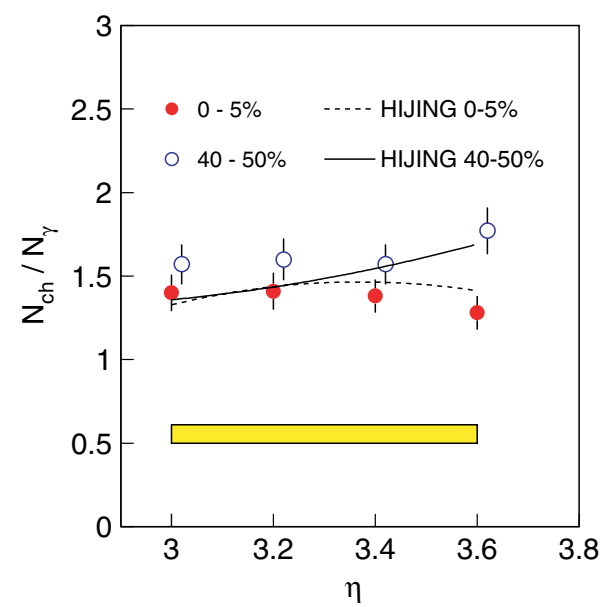

FIG. 12. (Color online) $N_{\mathrm{ch}} / N_{\gamma}$ for $0-5 \%$ and $40-50 \%$ central $\mathrm{Au}+\mathrm{Au}$ collisions at $\sqrt{s_{N N}}=62.4 \mathrm{GeV}$ as a function of $\eta$. Results from HIJING are also shown for comparison. The lower band reflects the common errors in ratio for the two centrality classes. 


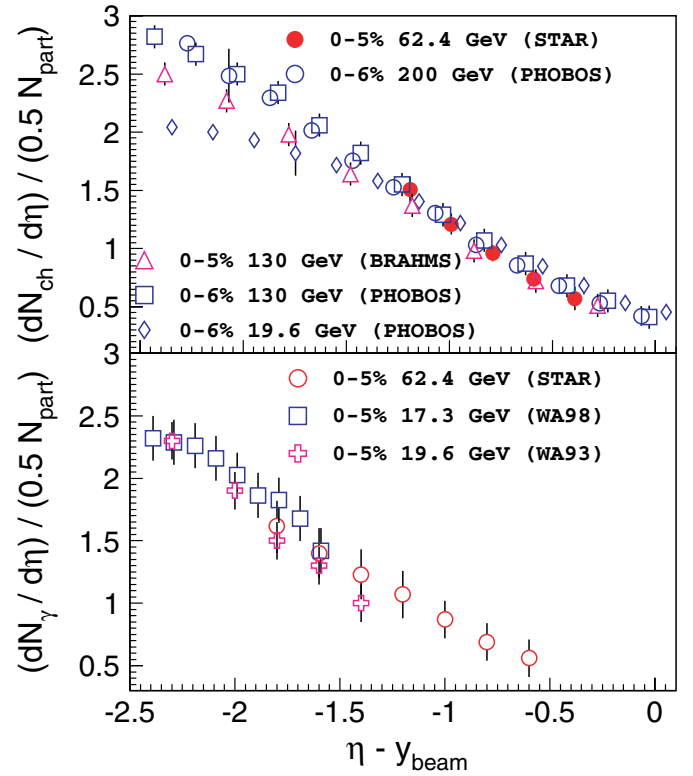

FIG. 13. (Color online) Top: variation of $d N_{\mathrm{ch}} / d \eta$ normalized to $N_{\text {part }}$ with $\eta-y_{\text {beam }}$ for different collision energies for central collisions. Bottom: variation of $d N_{\gamma} / d \eta$ normalized to $N_{\text {part }}$ with $\eta-$ $y_{\text {beam }}$ for different collision energies for central collisions.

\section{F. Energy dependence of limiting fragmentation}

Continuing our discussion on particle density in $\eta$, we now present results on the longitudinal scaling of particle production in heavy ion collisions. Here, we discuss the results on energy dependence of limiting fragmentation at $62.4 \mathrm{GeV}$ for charged particles and photons produced in $\mathrm{Au}+\mathrm{Au}$ collisions. In the subsequent sections we discuss the centrality and species dependence of this scaling.

In Fig. 13, we present the energy dependence of limiting fragmentation for inclusive charged particles and photons. The charged particle pseudorapidity distribution for central $(0-5 \%) \mathrm{Au}+\mathrm{Au}$ collisions at $\sqrt{s_{N N}}=62.4 \mathrm{GeV}$ is compared to the charged particle pseudorapidity distributions from PHOBOS for central $(0-6 \%)$ collisions at 19.6, 130, and $200 \mathrm{GeV}[8]$ and charged particle pseudorapidity distribution from BRAHMS for central (0-5\%) collisions at $130 \mathrm{GeV}$ [32]. The photon pseudorapidity distribution for central $(0-5 \%)$ $\mathrm{Au}+\mathrm{Au}$ collisions at $\sqrt{s_{N N}}=62.4 \mathrm{GeV}$ is compared with central $(0-5 \%)$ photon data for $\mathrm{Pb}+\mathrm{Pb}$ collisions at $17.3 \mathrm{GeV}$ from the WA98 experiment [29] and $19.6 \mathrm{GeV}$ central $(0-5 \%)$ $\mathrm{S}+\mathrm{Au}$ collision data from the WA93 experiment [35]. We observe in Fig. 13 that the SPS and RHIC $(62.4 \mathrm{GeV})$ photon results are consistent with each other, suggesting that photon production follows an energy-independent limiting fragmentation behavior. The charged particles at $62.4 \mathrm{GeV}$ also show an energy-independent limiting fragmentation behavior.

\section{G. Centrality dependence of limiting fragmentation}

Recently, contradictory results have been reported from inclusive charged particle measurements regarding the centrality dependence of the limiting fragmentation behavior. Results from PHOBOS show a centrality dependence [8]; those from
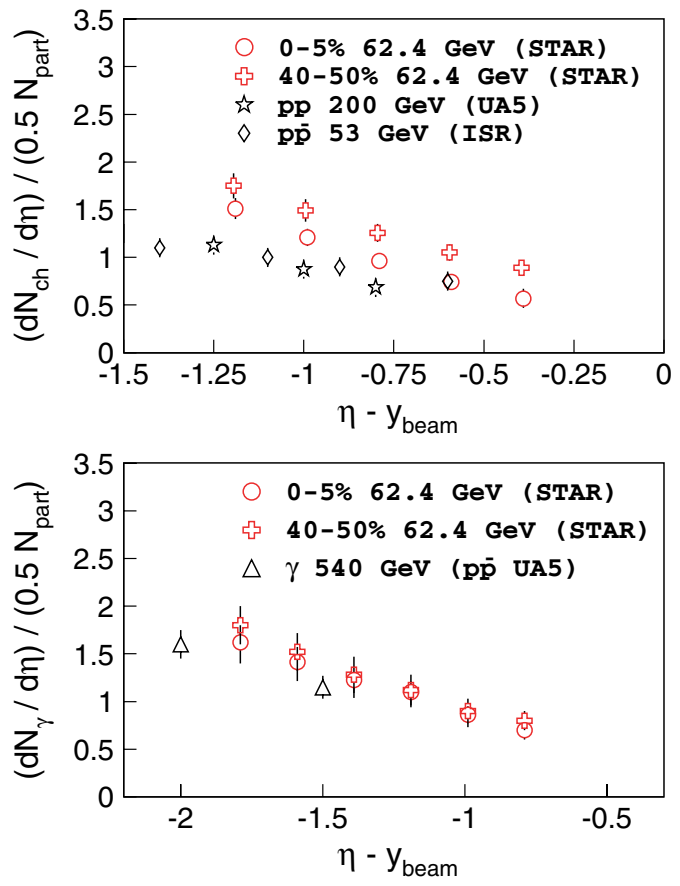

FIG. 14. (Color online) Top: variation of $d N_{\mathrm{ch}} / d \eta$ normalized to $N_{\text {part }}$ with $\eta-y_{\text {beam }}$ for central and peripheral collisions. Bottom: variation of $d N_{\gamma} / d \eta$ normalized to $N_{\text {part }}$ with $\eta-y_{\text {beam }}$ for central and peripheral collisions. Also shown are the charged particle and photon yields in $p+p$ and $p+\bar{p}$ collisions.

BRAHMS show a centrality independent behavior [32]. Here, we present the results on the centrality dependence of limiting fragmentation for charged particles and photons at $\sqrt{s_{N N}}=$ $62.4 \mathrm{GeV}$.

In Fig. 14, we show the centrality dependence of limiting fragmentation for charged particles and photons. The charged particle pseudorapidity distributions for $0-5 \%$ and $40-50 \%$ central $\mathrm{Au}+\mathrm{Au}$ collisions at $\sqrt{s_{N N}}=62.4 \mathrm{GeV}$ have been compared. We observe, at forward rapidity, the charged particle yield normalized to the number of participating nucleons as a function of $\eta-y_{\text {beam }}$ is higher for peripheral collisions compared to central collisions, whereas within the measured $\eta$ range of 2.3-3.7, the photon yield normalized to the number of participating nucleons as a function of $\eta-y_{\text {beam }}$ is found to be independent of centrality. The dependence of limiting fragmentation on the collision system is most clearly seen in the comparison between results from heavy ion collisions with those from $p+p$ and $p+\bar{p}$ collisions [36]. We observe in Fig. 14 that the photon results in the forward rapidity region from $p \bar{p}$ collisions at $\sqrt{s_{N N}}=540 \mathrm{GeV}$ are in close agreement with the measured photon yield in $\mathrm{Au}+\mathrm{Au}$ collisions at $\sqrt{s_{N N}}=62.4 \mathrm{GeV}$. However the $p+p$ and $p+\bar{p}$ inclusive charged particle results are very different from those for $\mathrm{Au}+\mathrm{Au}$ collisions at $\sqrt{s_{N N}}=62.4 \mathrm{GeV}$. It may be mentioned that the photon yield is dominated by photons from decay of $\pi^{0} \mathrm{~s}$ [10]. The presented photon results and their comparison with nucleon-nucleon collisions indicate that in the $\eta$ region studied, there is apparently a significant charged baryon contribution in nucleus-nucleus collisions. Similar 


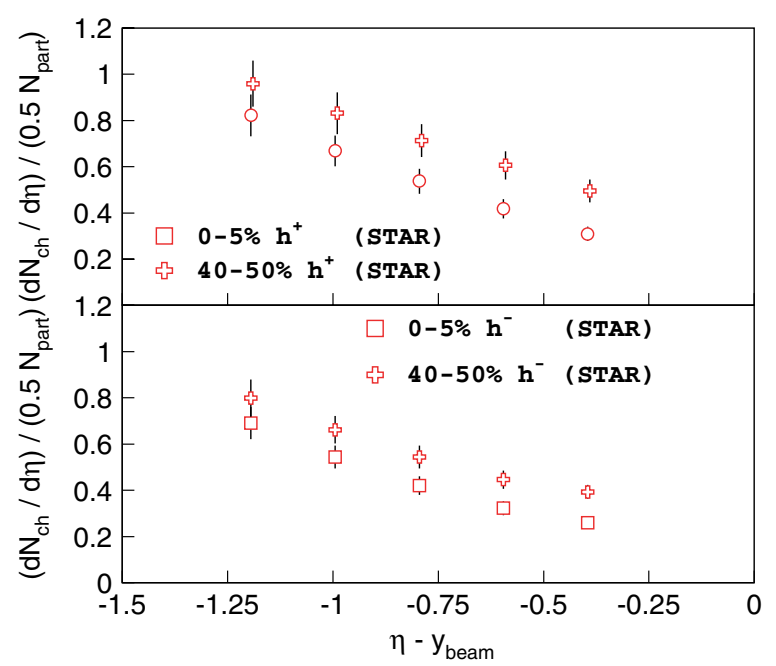

FIG. 15. (Color online) Variation of $d N_{\mathrm{ch}} / d \eta$ normalized to $N_{\text {part }}$ with $\eta-y_{\text {beam }}$ for central and peripheral collisions for positively $\left(h^{+}\right)$ and negatively charged $\left(h^{-}\right)$hadrons.

centrality-dependent behavior of limiting fragmentation for charged particles was also observed by PHOBOS [8]. The centrality dependence of limiting fragmentation in charged particles has been speculated to be due to nuclear remnants and baryon stopping $[8,12]$. The centrality-independent limiting fragmentation for photons has been attributed to mesons being the dominant source of photon production [10]. HIJING calculations indicate that about $93-96 \%$ of the photons are from $\pi^{0}$ decay.

In order to understand the role of nuclear remnants and baryon stopping in the observed centrality-dependent behavior of limiting fragmentation of charged particles, we studied separately the limiting fragmentation for positively and negatively charged hadrons. The contribution from protons coming from beam remnants can be understood by studying the limiting fragmentation of positively charged hadrons. In Fig. 15, we plotted $d N_{\mathrm{ch}} / d \eta$ normalized to the number of participating nucleons for $40-50 \%$ and for $0-5 \%$ central collisions for positively $\left(h^{+}\right)$and negatively charged $\left(h^{-}\right)$hadrons. In addition to the systematic errors discussed earlier, and shown in the figure, there is an error due to the uncertainty in the charge determination. The uncertainty has been studied by embedding charged Monte Carlo tracks into real data and then following the full reconstruction chain. This error was obtained as a function of $\eta$ and is defined as the ratio of the total number of embedded charged tracks whose charge has been reconstructed incorrectly, to the total number of charged tracks embedded. The error in charge determination was found to increase from $2 \%$ at $\eta=2.9$ to $15 \%$ at $\eta=3.9$. Both $h^{+}$and $h^{-}$show a centrality-dependent limiting fragmentation behavior. When compared to the centrality-independent limiting fragmentation behavior for photons (Fig. 14) and to results from nucleonnucleon collisions (Fig. 14), our measurements indicate that baryon transport at forward rapidity also plays an important role in the observed centrality-dependent behavior of limiting fragmentation for charged particles. We find that the ratio for yields of $h^{+}$from peripheral to central collisions increases from $1.17 \pm 0.06$ at $\eta=3.0$ to $1.61 \pm 0.07$ at $\eta=3.8$ (closer to beam rapidity). The values for $h^{-}$are $1.16 \pm 0.06$ at $\eta=3.0$ and $1.51 \pm 0.07$ at $\eta=3.8$. From these values, we find that the increase in the ratio with $\eta$ seems to be somewhat weaker for $h^{-}$compared to $h^{+}$. However, within the systematic errors, it is difficult to determine the role of the beam remnants (beam protons in $h^{+}$) in the centrality-dependent behavior of limiting fragmentation for charged particles at forward rapidity.

Energy and centrality dependence of limiting fragmentation for charged particles can be a test for particle production models. We have observed that particle production models such as HIJING and AMPT reasonably describe the $\eta$ distribution of charged particles at forward rapidity. Now we investigate whether they can qualitatively reproduce the limiting fragmentation features of experimental data. Our calculations show that in the HIJING and AMPT models, the charged particles show energy-independent limiting fragmentation. The centrality-dependent behavior of limiting fragmentation for charged particles is more clearly observed in the AMPT model than in the HIJING. In Fig. 16, we only show the results from the AMPT model. These results are for the $\sqrt{s_{N N}}$ values of 19.6, 62.4, and $130 \mathrm{GeV} \mathrm{Au+Au}$ collisions at $0-3$ and 9-12 fm impact parameters. For the centrality dependence, we only show the results for $\sqrt{s_{N N}}=19.6 \mathrm{GeV}$, the energy at which the centrality-dependent effect is most prominent in the data [8]. The AMPT model has qualitative limiting
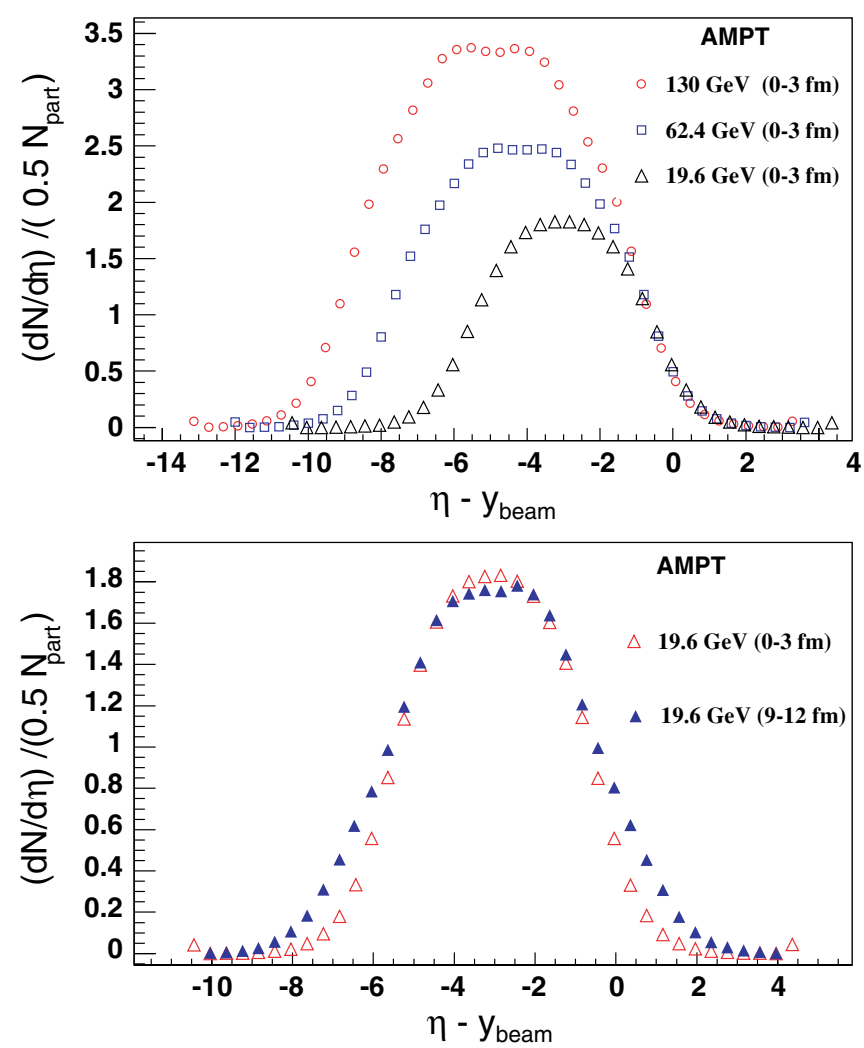

FIG. 16. (Color online) Variation of $d N_{\mathrm{ch}} / d \eta$ normalized to $N_{\text {part }}$ with $\eta-y_{\text {beam }}$ from AMPT model [22] calculations for various c.m. energies in central collisions (top) and central and peripheral collisions at $\sqrt{s_{N N}}=19.6 \mathrm{GeV}$ (bottom). 
fragmentation features similar to those of experimental data (shown in Fig. 14). We find in the model that the central yields, when normalized to the number of particpating nucleons, are also lower than the corresponding peripheral yields at forward rapidity when $\eta$ is shifted by the beam rapidity.

\section{H. Identified particle limiting fragmentation}

The observation of centrality-dependent and energyindependent limiting fragmentation for inclusive charged particles, along with the centrality- and energy-independent limiting fragmentation for photons (presented in previous sections), motivates us to study the limiting fragmentation of identified particles.

The top panel in Fig. 17 shows the charged pion rapidity density in central $\mathrm{Au}+\mathrm{Au}$ collisions at $\mathrm{RHIC}$ [37], $\mathrm{Pb}+\mathrm{Pb}$ collisions at the SPS [38], and $\mathrm{Au}+\mathrm{Au}$ collisions at the alternating-current synchroton (AGS) [3]. Also shown is the estimated $\pi^{0}$ rapidity density from the present measurement of the photon rapidity density at $\sqrt{s_{N N}}=62.4 \mathrm{GeV}$, all
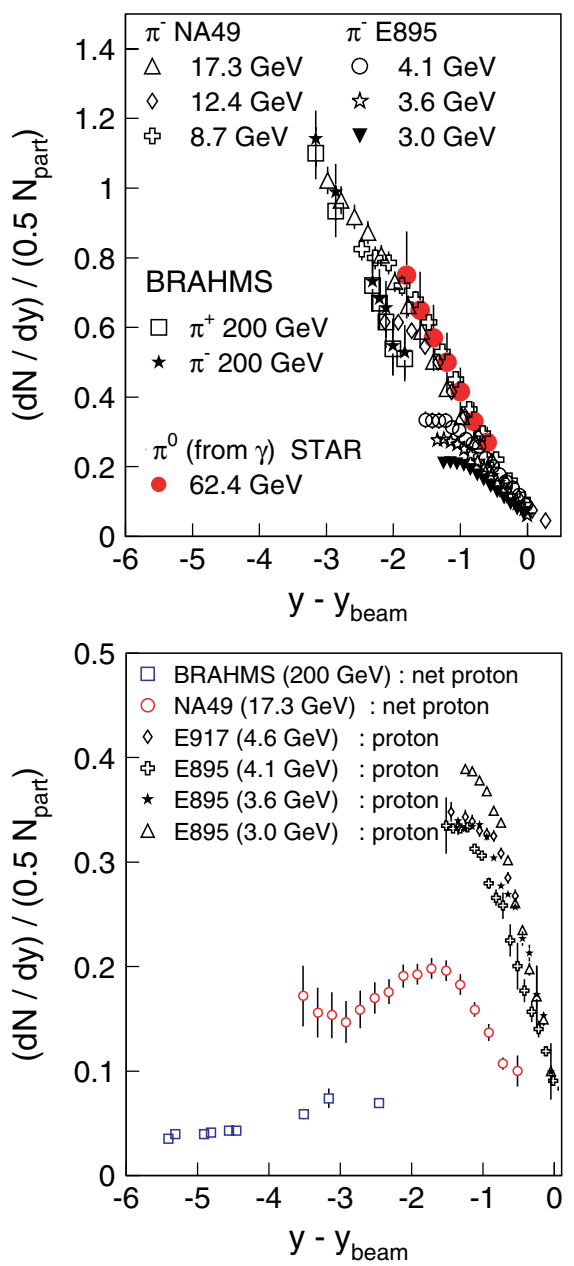

FIG. 17. (Color online) Top: variation of pion rapidity density normalized to $N_{\text {part }}$ with $y-y_{\text {beam }}$ for central collisions at various collision energies. Also shown is the estimated $d N_{\pi^{0}} / d y$ obtained from $d N_{\gamma} / d y$ normalized to $N_{\text {part }}$. Bottom: variation of net proton rapidity density normalized to $N_{\text {part }}$ with $y-y_{\text {beam }}$ for central collisions at various collision energies. as a function of $y-y_{\text {beam }}[10]$. We obtained the ratio of the photon to $\pi^{0}$ yields from HIJING. This ratio is used to estimate the $\pi^{0}$ yield from the measured photon yield. The results indicate that pion production in heavy ion collisions in the fragmentation region agrees with the energy-independent limiting fragmentation picture.

The bottom panel of Fig. 17 shows the net proton $(p-\bar{p})$ rapidity density in central $\mathrm{Au}+\mathrm{Au}$ collisions at RHIC [34] energies and $\mathrm{Pb}+\mathrm{Pb}$ collisions at SPS [39] energies. For AGS energies $[3,40]$, we plot only the proton rapidity density in $\mathrm{Au}+\mathrm{Au}$ collisions. Since the antiproton yields are very low ( $\bar{p} / p \sim 2 \times 10^{-4}$ at top AGS energy), the proton rapidity density reflects the net proton rapidity distribution. The net protons violate the energy dependence of limiting fragmentation. These results show that baryons and mesons differ in the energy dependence of limiting fragmentation. The results for identified particles, along with the centrality dependence of limiting fragmentation for inclusive charged hadrons, and the centrality independence of limiting fragmentation for identified mesons, shows that the baryon transport in heavy ion collisions plays an important role in particle production at forward rapidity. The results also show that although baryon stopping is different in different collision systems, the pions produced at forward rapidity are not affected by baryon transport. The limiting fragmentation study for net protons may also indicate the validity of a baryon junction picture [12]. If the baryon numbers are carried by the valence quarks, then at forward rapidity the baryons should also follow an energy-independent limiting fragmentation behavior, like pions (originating from valence quarks). This may indicate that the baryon number is not carried by the valence quark, which is suggested in the baryon junction picture, where the baryon number resides in a nonperturbative configuration of gluon fields, rather than in the valence quarks.

\section{SUMMARY}

In summary, we have presented charged particle and photon multiplicity measurements at RHIC in the pseudorapidity regions $2.9 \leqslant \eta \leqslant 3.9$ and $2.3 \leqslant \eta \leqslant 3.7$, respectively. The pseudorapidity distributions of charged particles and photons for $\mathrm{Au}+\mathrm{Au}$ collisions at $\sqrt{s_{N N}}=62.4 \mathrm{GeV}$ have been obtained for various centrality classes and compared to results from different models. Charged particle and photon production normalized to the number of participating nucleon pairs and to the number of binary collisions has been studied. The photon multiplicity, within the systematic errors, seems to scale with the number of participating nucleons, while the charged particle multiplicity does not. Both the photon and charged particle production at forward rapidity do not scale with number of binary collisions. This indicates that the particle production at forward rapidity is not dominated by a contribution from hard processes. Charged particle and photon distributions at $\sqrt{s_{N N}}=62.4 \mathrm{GeV}$ are both observed to be consistent with the energy-independent limiting fragmentation scenario. Photon production is observed to follow a centrality-independent limiting fragmentation scenario, while the charged particles follow a centrality-dependent behavior. Comparison of the pseudorapidity distributions of positively charged particles, 
negatively charged particles, pions, and distributions from $p+p$ collisions indicates that the baryons are responsible for the centrality-dependent limiting fragmentation behavior of charged particles. The study of limiting fragmentation for pions and net protons shows that mesons follow energyindependent limiting fragmentation, whereas baryons do not.

\section{ACKNOWLEDGMENTS}

We thank S. Jeon for providing us the LEXUS results for comparison and G. Veres for providing us the NA49 data on net protons. We thank the RHIC Operations Group and RCF at BNL, and the NERSC Center at LBNL for their support. We acknowledge the help of CERN for use of GASSIPLEX chips in the PMD readout. This work was supported in part by the HENP Divisions of the Office of Science of the U.S. DOE; the U.S. NSF; the BMBF of Germany; IN2P3, RA, RPL, and EMN of France; EPSRC of the United Kingdom; FAPESP of Brazil; the Russian Ministry of Science and Technology; the Ministry of Education and the NNSFC of China; SFOM of the Czech Republic, FOM and UU of the Netherlands; DAE, DST, and CSIR of the Government of India; the Swiss NSF; the Polish State Committee for Scientific Research; STAA of Slovakia, and the Korea Sci. \& Eng. Foundation.
[1] K. H. Ackermann et al., Nucl. Instrum. Methods A 499, 624 (2003).

[2] J. D. Bjorken, Phys. Rev. D 27, 140 (1983); L. D. Landau, Izv. Akad. Nauk SSSR Ser. Fiz. 17, 51 (1953); S. Belenkij and L. D. Landau, Usp. Fiz. Nauk 56, 309 (1955); Nuovo Cimento Suppl. 3S10, 15 (1956); R. C. Hwa and K. Kajantie, Phys. Rev. D 32, 1109 (1985); J. Alam et al., Ann. Phys. (NY) 286, 159 (2000); B. Mohanty and Jan-e Alam, Phys. Rev. C 68, 064903 (2003).

[3] J. L. Klay et al. (E895 Collaboration), Phys. Rev. Lett. 88, 102301 (2002); J. L. Klay et al. (E895 Collaboration), Phys. Rev. C 68, 054905 (2003).

[4] P. K. Netrakanti and B. Mohanty, Phys. Rev. C 71, 047901 (2005); J. Aichelin and K. Werner, Phys. Lett. B300, 158 (1993).

[5] L. V. Gribov, E. M. Levin, and M. G. Ryskin, Phys. Rep. 100, 1 (1983); J. P. Blaizot and A. H. Mueller, Nucl. Phys. B289, 847 (1987)

[6] L. McLerran and R. Venugopalan, Phys. Rev. D 49, 2233 (1994); 50, 2225 (1994); Y. V. Kovchegov, ibid. 54, 5463 (1996); E. Iancu and L. McLerran, Phys. Lett. B510, 145 (2001); A. Krasnitz and R. Venugopalan, Phys. Rev. Lett. 84, 4309 (2000).

[7] K. Adcox et al. (PHENIX Collaboration), Phys. Rev. Lett. 86, 3500 (2001).

[8] B. B. Back et al. (PHOBOS Collaboration), Phys. Rev. Lett. 87, 102303 (2001); 91, 052303 (2003).

[9] D. Kharzeev, E. Levin, and M. Nardi, Phys. Rev. C 71, 054903 (2005).

[10] J. Adams et al. (STAR Collaboration), Phys. Rev. Lett. 95, 062301 (2005).

[11] J. Benecke et al., Phys. Rev. 188, 2159 (1969); R. Beckmann, S. Raha, N. Stelte, and R. M. Weiner, Phys. Lett. B105, 411 (1981).

[12] D. Kharzeev, Phys. Lett. B378, 238 (1996); V. T. Pop, M. Gyulassy, J. Barrette, C. Gale, X. N. Wang, and N. Xu, Phys. Rev. C 70, 064906 (2004).

[13] K. Itakura, Y. V. Kovchegov, L. McLerran, and D. Teaney, Nucl. Phys. A730, 160 (2004).

[14] J. Jalilian-Marian, Phys. Rev. C 70, 027902 (2004).

[15] S. Jeon, V. T. Pop, and M. Bleicher, Phys. Rev. C 69, 044904 (2004).

[16] M. M. Aggarwal et al. (WA98 Collaboration), Phys. Rev. C 65, 054912 (2002).

[17] H. Heiselberg, Phys. Rep. 351, 161 (2001).

[18] B. Mohanty and J. Serreau, Phys. Rep. 414, 263 (2005); M. M. Aggarwal et al. (WA98 Collaboration), Phys. Rev. C 64, 011901(R) (2001).
[19] K. H. Ackermann et al., Nucl. Instrum. Methods A 499, 713 (2003).

[20] M. M. Aggarwal et al., Nucl. Instrum. Methods A 499, 751 (2003); 488, 131 (2002).

[21] X.-N. Wang and M. Gyulassy, Phys. Rev. D 44, 3501 (1991).

[22] B. Zhang, C. M. Ko, B. A. Li, and Z. Lin, Phys. Rev. C 61, 067901 (2000)

[23] F. S. Bieser et al., Nucl. Instrum. Methods A 499, 766 (2003).

[24] J. Adams et al. (STAR Collaboration), nucl-ex/0311017.

[25] J. Putschke, Ph.D. thesis, Max-Planck-Institut für Physik, Munich, 2004; F. Simon, Ph.D. thesis, Max-Planck-Institut für Physik, Munich, 2004.

[26] M. Oldenburg, Ph.D. thesis, Max-Planck-Institut für Physik, Munich, 2002.

[27] C. Adler et al. (STAR Collaboration), Phys. Rev. Lett. 87, 112303 (2001).

[28] V. Fine and P. Nevski, Published in Proceedings of International Conference On Computing In High-Energy Physics And Nuclear Physics (CHEP 2000), Padova, Italy, edited by Mirco Mazzucato and Michele Michelotto, 2001, p. 763.

[29] M. M. Aggarwal et al. (WA98 Collaboration), Phys. Lett. B458, 422 (1999)

[30] S. Jeon and J. Kapusta, Phys. Rev. C 56, 468 (1997).

[31] W. Thome et al., Nucl. Phys. B129, 365 (1977).

[32] I. G. Bearden et al. (BRAHMS Collaboration), Phys. Lett. B523, 227 (2001); I. G. Bearden et al. (BRAHMS Collaboration), Phys. Rev. Lett. 88, 202301 (2002).

[33] D. Kharzeev and M. Nardi, Phys. Lett. B507, 121 (2001); D. Kharzeev and E. Levin, ibid. B523, 79 (2001).

[34] I. G. Bearden et al. (BRAHMS Collaboration), Phys. Rev. Lett. 93, 102301 (2004).

[35] M. M. Aggarwal et al. (WA93 Collaboration), Phys. Rev. C 58, 1146 (1998); Phys. Lett. B403, 390 (1997).

[36] K. Alpgard et al. (UA5 Collaboration), Phys. Lett. B115, 71 (1982); G. J. Alner et al., Z. Phys. C 33, 1 (1986).

[37] I. G. Bearden et al. (BRAHMS Collaboration), Phys. Rev. Lett. 94, 162301 (2005).

[38] S. V. Afanasiev et al. (NA49 Collaboration), Phys. Rev. C 66, 054902 (2002).

[39] H. Appelshäuser et al. (NA49 Collaboration), Phys. Rev. Lett. 82, 2471 (1999).

[40] B. B. Back et al. (E917 Collaboration), Phys. Rev. C 66, 054901 (2002). 